\title{
Temporal and inter-specific variations in forage fish feeding conditions in the NW Mediterranean: lipid content and fatty acid compositional changes
}

\author{
H. Pethybridge ${ }^{1}$, N. Bodin ${ }^{2,3, *}$, E.-J. Arsenault-Pernet ${ }^{3}$, J.-H. Bourdeix ${ }^{4}$, B. Brisset ${ }^{4}$, \\ J.-L. Bigot ${ }^{4}$, D. $\operatorname{Roos}^{4}$, M. Peter \\ ${ }^{1}$ CSIRO Marine and Atmospheric Research, GPO Box 1538, Hobart, Tasmania 7000, Australia \\ ${ }^{2}$ IRD, UMR 212 EME - Fishing Port, PO Box 570, Victoria, Mahé, Seychelles \\ ${ }^{3}$ IRD, UMR 212 EME - Avenue Jean Monnet, BP 70, 34200 Sète, France \\ ${ }^{4}$ IFREMER, UMR 212 EME - Avenue Jean Monnet, BP 70, 34200 Sète, France \\ ${ }^{5}$ Ecole d'Ingénieurs de Purpan, 75 voie du Toec, BP 57611, 31076 Toulouse Cedex 3, France
}

\begin{abstract}
We describe the total lipid content, lipid class composition and fatty acid profiles of adult forage fishes (anchovy, sardine and sprat) sampled in the NW Mediterranean Sea in 2010 and 2011. Inter- and intra-species differences were mostly related to sampling period with limited effect of gender or total length. As an assemblage, total lipid content and relative levels of triacylglycerols and fatty acids 16:1n7, 20:5n3 and 14:0 in forage fish were highest in summer and autumn, indicating better feeding conditions and a more pronounced diatom-supported food web. In contrast, total lipid content was lowest at the end of winter and spring, and coincided with high levels of 22:6n3, indicating a more herbivorous diet based on dinoflagellates. Resource partitioning and niche separation, as inferred from fatty acid profiles, were apparent between species. Sardine showed a more diverse, temporally separated feeding strategy than anchovy, and dietary overlap was higher in winter than summer with sardine having higher markers of copepods, 22:1n11 and 20:1n9. Sprat collected in winter occupied a separate niche area to both sardine and anchovy with higher total lipid content and carnivory biomarker 18:1n9. Our results show that the lipid dynamics of forage fishes can be used to gain quantitative insights into sub-system level changes in species interactions, including prey and predator productivity.
\end{abstract}

KEY WORDS: Trophic markers · Environmental variations $\cdot$ Clupeiformes $\cdot$ Nutritional condition · Prey quality $\cdot$ Ecosystem health

\section{INTRODUCTION}

Foraging fishes (of the families Clupeidae and Engraulidae) play a fundamental role in marine trophodynamics: they uptake the energy available from low-level plankton and provide top-order predators, including marine mammals, seabirds, large piscivorous fishes and humans, with a highly nutritious and energetic food source (Cury et al. 2000). Owing to the resulting bottom-up and top-down (wasp-waist ecosystem) constraints, in addition to fishing pressures and an $r$-strategy life-history, the population dynamics of mid-trophic fishes are subject to considerable fluctuations caused by environmental variability (Lloret et al. 2004, Santojanni et al. 2006, Engelhard et al. 2014). The close relationship between food supply, individual performance and population dynamics of forage fishes (Daskalov 2002, Pikitch et al. 2012, Pethybridge et al. 2013a) emphasizes the need to better understand their feeding 
ecology, including prey availability (quality and quantity). Furthermore, the importance of forage fishes to top-order predators and humans necessitates that information on their nutritive and energetic quality is understood and reported.

In the NW Mediterranean Sea, European anchovy Engraulis encrasicolus and sardine Sardina pilchardus are the most abundant and commercially important forage fishes, with sprat Sprattus sprattus also well represented in the region (Palomera et al. 2007). The Gulf of Lions (GoL) is one of the most important spawning areas for these species (García \& Palomera 1996). Climate and ecosystem variability in the GoL operate over a range of spatial and temporal scales that affect phytoplankton biomass and production (Gaudy et al. 2003, Siokou-Frangou et al. 2010). Both temperature and primary production follow annual cycles characterized by strong seasonality (Razouls \& Kouwenberg 1993, Lazzari et al. 2011). Forage fishes in this region are classified as omnivores, having trophic positions between 2.2 and 3.7 (Plounevez \& Champalbert 2000, Stergiou \& Karpouzi 2001, Costalago et al. 2012). Based on gut content analysis, sardines are thought to be dominant consumers of small zooplankton, while anchovy and sprat consume larger zooplankton with a high presence of copepods all year round (Tudela \& Palomera 1997, Plounevez \& Champalbert 2000). Although important taxonomic and baseline data can be obtained through gut content analysis, difficulties associated with identifying planktonic material, underrepresentation of small and/or soft prey items and providing only a snap-shot representation of diet are well known impediments. In response, the application of a range of biochemical tracer techniques is increasing due to their ability to examine functional properties and life-history strategies at the organism level with ecosystem-scale patterns.

Lipids and their components (classes and fatty acids) are important macronutrients for energy storage in marine fish (Tocher 2003, Parrish 2013) and serve a diverse range of functions including structural integrity of cells and bioeffector roles. They can be used to indicate anaerobic (energy gain) and catabolic (energy loss) processes central to an organism's fitness (Adams 1999). As the degree of lipid accumulation depends on the supply of dietary fat, measurements of lipids and their components can be correlated with changes in ecosystem function (e.g. energy fluxes, Goncalves et al. 2012; and primary productivity, Pethybridge et al. 2013b). They can also be related to structural changes in an ecosystem as many are transferred with limited modification from one trophic level to the next (Dalsgaard et al. 2003, Iverson et al. 2004), allowing them to be ideal tracers of trophic relationships. Furthermore, the reorganisation of fish communities or regime shifts can be linked to the availability of essential fatty acids (Litzow et al. 2006). As foraging fishes are the main energy transfer agents in pelagic ecosystems, assessing their lipid dynamics can generate system-level understanding of marine trophodynamics. For example, seasonal lipid dynamics of herring and sprat in the Baltic Sea have been related to local feeding conditions and inter-specific differences in diets and reproductive strategies (Røjbek et al. 2014). Furthermore, long-term, interannual (1960 to 2001) changes in sprat lipid content (\% body fat) in the Black Sea were correlated with stock biomass and phytoplankton biomass (Shulman et al. 2005), demonstrating the potential for total lipid content to be used as an indicator for stock assessments and local feeding conditions.

The ability to detect and monitor large-scale changes in ecosystem functioning is urgent, as many species including forage fishes are likely to be highly sensitive to the predicted impacts of climate change, particularly in climate 'hotspot' areas such as the Mediterranean Sea (Giorgi \& Lionello 2008). In this study, lipid and fatty acid tracers were used to explore intraand inter-species variations in the diet, nutritional condition and energy allocation strategies of 3 forage fishes from the GoL (NW Mediterranean Sea). Of particular interest was testing for tissue-related, temporal and spatial as well as between-species differences. For the fatty acids, we took a novel approach in analysing the composition of the neutral (as opposed to polar or total) lipid fraction as it is more representative of dietary than physiology components. Such a comparative approach provides valuable insights into how these sympatric fishes partition space and prey resources.

\section{MATERIALS AND METHODS}

\section{Study area and sampling}

The study area was located in the GoL, a large continental shelf located in the NW Mediterranean Sea (between $42^{\circ} 15^{\prime}$ to $43^{\circ} 35^{\prime} \mathrm{N}$, and $3^{\circ} 00^{\prime}$ to $6^{\circ} 00^{\prime} \mathrm{E}$; Fig. 1). A total of 116 fishes were collected in the coastal and offshore waters in the east and west areas of the GoL in July 2010 and March, May and November 2011 (see Table 1). July samples were obtained during research surveys (PELMED10) conducted on 


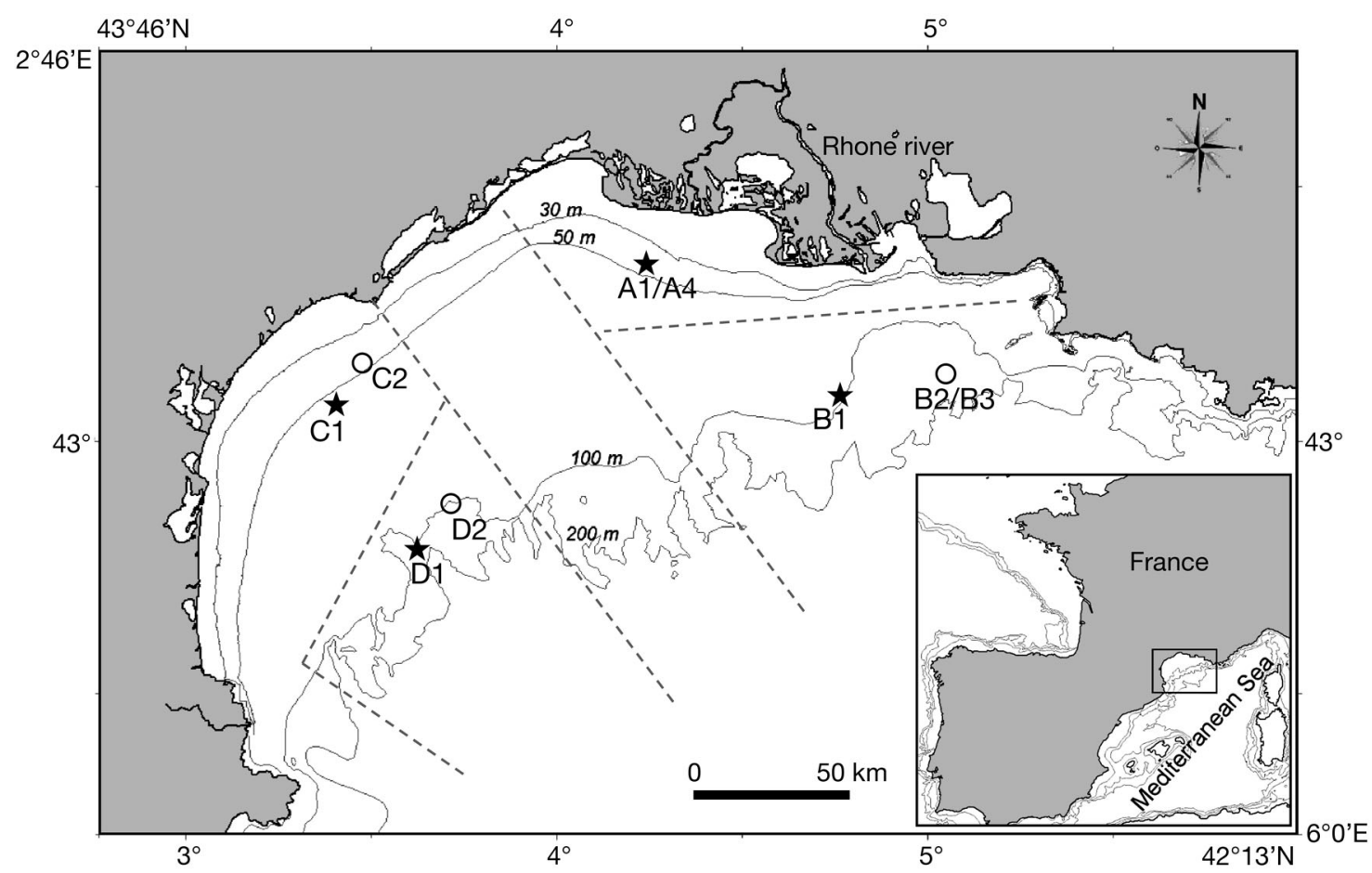

Fig. 1. Collection areas within the Gulf of Lions, NW Mediterranean Sea. Sites A1 and A4: east and coastal sites sampled in July 2010 and November 2011; B1, B2 and B3: east and slope sites sampled in July 2010, March 2011 and May 2011 ; C1 and C2: west and coastal sites sampled in July 2010 and March 2011; D1 and D2: west and slope sites sampled in July 2010 and March 2011

board the RV 'L'Europe' and led by IFREMER, France. March, May and November samples were obtained on board the fishing trawl vessel 'Roger-Christian III' (Sète, France). Upon collection, all specimens were identified taxonomically, measured, sexed and assigned a maturity stage based on macroscopic determination of oocytes. Samples were then immediately dissected and sub-samples of muscle and liver were collected and stored at $-80^{\circ} \mathrm{C}$ until further analysis.

\section{Lipid and fatty acid analyses}

A sub-sample (approximately $0.1 \pm 0.001 \mathrm{~g}$ ) of each tissue was weighted under nitrogen gas, and extracted according to Folch et al. (1957). Briefly, $4 \mathrm{ml}$ of dichloromethane:methanol solvent mixture (2:1 $\mathrm{v} / \mathrm{v})$ were added, and lipids were extracted with a potter homogenizer (glass/Teflon) at $0^{\circ} \mathrm{C}$. After addition of $1 \mathrm{ml}$ of aqueous $\mathrm{KCl}(0.88 \% \mathrm{w} / \mathrm{v})$, the extract was further vortexed and centrifuged at $2000 \mathrm{rpm}$ $(\mathrm{rcf}=850)$ for $2 \mathrm{~min}$, allowing for the recovery of total lipids from the lower organic phase. The total lipid extract (TLE) was concentrated with a RapidVap ${ }^{\circledR}$ vacuum at $35^{\circ} \mathrm{C}$ (Labconco, Fisher Scientific) and diluted with dichloromethane for further analysis.
Lipid classes were quantified using an Iatroscan MK-VI (Iatron Laboratories) thin-layer chromatography-flame ionization detector analyzer (TLCFID). An aliquot of TLE was spotted on quartz chromarods SIII covered with silica, and separated into triacylglycerols (TAG), free sterols (ST), sterol- and wax-esters (SE-WE), ketones (KET), free fatty acids (FFA), acetone mobile polar lipids (AMPL) and phospholipids (PL). Concentrations of lipid classes were expressed in $\mathrm{Mg} \mathrm{mg}^{-1}$ on a wet weight (ww) basis using suitable calibration curves. Total lipid content $\left(\mu \mathrm{g} \mathrm{mg}{ }^{-1} \mathrm{ww}\right.$ ) corresponds to the sum of lipid classes, and was used to calculate the relative contribution (\%) of each lipid class to the total fat. Replicate measurements of internal reference material (tuna muscle and liver) determined that the analytical variability of iatroscan-produced results was $\pm 12 \%$.

For fatty acids, a second aliquot of the TLE was further separated by adsorption chromatography on a silica gel micro-column (Kieselgel 70 to 230 mesh, heated at $450^{\circ} \mathrm{C}$ and deactivated with $6 \%$ water). The neutral lipids were eluted with $10 \mathrm{ml}$ chloroform:methanol mixture $(98: 2 \mathrm{v} / \mathrm{v})$. A known amount of 23:0 fatty acid was added as an internal standard. Neutral lipid fractions were transmethylated at $100^{\circ} \mathrm{C}$ with $10 \mathrm{wt} \%$ boron trifluoride-methanol (Met- 
calfe \& Schmitz 1961). The fatty acid methyl esters were separated and quantified by gas chromatography (Clarus 600, Perkin-Elmer) using a capillary column (Omegawax 100, $15 \mathrm{~m} \times 0.10 \mathrm{~mm}$ i.d., $0.10 \mu \mathrm{m}$ film, Supelco) and a flame ionization detector. Helium $\left(0.5 \mathrm{ml} \mathrm{min}^{-1}\right)$ was used as carrier gas and temperature programming was from 160 to $200^{\circ} \mathrm{C}$ at $20^{\circ} \mathrm{C} \mathrm{min}^{-1}$ and then to $270^{\circ} \mathrm{C}$ at $30^{\circ} \mathrm{C} \mathrm{min}^{-1}$. Individual fatty acid methyl esters were identified by comparison to known standards (PUFA 1 marine source, PUFA 3 menhaden oil, Supelco).

\section{Indices of nutritional condition and trophic relationships}

Total lipid content, calculated ratios of triacylglycerol to sterols (TAG/ST), and relative levels of TAG and essential fatty acids (22:6n3, 20:5n3 and 20:4n6) were used as descriptive indices of fish nutritional condition (or fitness) and local food quality and availability (Adams 1999, Weber et al. 2003). The lipid classes SE-WE and TAG are indicators of long- and short-term energy storage, respectively, and FFA an indicator of sample degradation.

To help assess broad scale food web groupings, a number of established fatty acid trophic markers were calculated. Known markers of diatoms include 14:0, 16:1n7, 18:1n 7 and 20:5n3, while markers of dinoflagellates include 22:6n3, 18:4n 3 and 22:5n3 (Dalsgaard et al. 2003, Parrish 2013). Relative ratios provide an indication of long-term trophic exchanges: for example, ratios of 16:1n7/16:0 and 20:5n3/22:6n 3 were used to discriminate between diatom and dinoflagellate feeding (Auel et al. 2002, Rossi et al. 2006). In particular, ratios of 16:1n7/16:0 > 2 are considered to represent a strong presence of diatoms whereas ratios < 0.3 suggest dinoflagellates; ratios of $18: 1 \mathrm{n} 9 /$ $18: 1 \mathrm{n} 7$ and $20: 5 \mathrm{n} 3 / 22: 6 \mathrm{n} 3$ are used as carnivory indices (Dalsgaard et al. 2003) and have been significantly correlated with stable isotopes of nitrogen (and thus, trophic position) and carbon (El Sabaawi et al. 2009). Typical algal markers consist of 18:3n3, 18:2n6 and 20:4n6 (Richoux 2011), while adult euphausiids (>15 mm, i.e Euphausia superb and Meganyctiphanes norvegica) are characterised by $20: 5 \mathrm{n} 3$ and 22:6n3 (Mayzaud et al. 1999). Large calanoid copepods (>4.1 mm, e.g. Calanus spp.) are often characterised by high levels of 22:1n11 and 20:1n9 (Dalsgaard et al. 2003), while smaller copepods from the Oncaea and Oithona genera have been shown to have high levels of 18:1n9, 16:0 and 20:5n3 (Kattner et al. 2003).

\section{Statistical analysis}

Differences in total lipid content and individual lipid classes between species, tissues, sampling location and month were tested by 1-way ANOVA, using SPSS statistical software v.17. Normality and homogeneity were verified using Kolmogorov-Smirnov and Levene tests.

For multivariate fatty acid data, Bray-Curtis similarity matrices were calculated for square-root transformed percent composition data to test the differences between various factors. Principal component analyses (PCA) and multidimensional scaling ordinations (MDS) were used to visually assess groupings within the dataset and identify the main fatty acids responsible for differences. Non parametric, permutational multivariate analysis of variance (PERMANOVA) was used to test for inter- and intra-specific differences and estimate components of variation due to sampling month (Mo, fixed with 3 to 4 levels), species (Sp, fixed with 2 levels), tissues (Ti, fixed with 2 levels), and zone (Zo, fixed with 2 levels). The effect of sex was only tested for anchovy in July and was found to be non-significant (PERMANOVA, $t=1.2$, $\mathrm{p}=0.078$ ). Total length was included as a covariate in PERMANOVAs but was shown to be non-significant in both sardine and anchovy separately, and for all forage fishes combined (including sprat). Due to occasional small within-group sample sizes, significant $\mathrm{p}$-values were obtained using Monte Carlo 999 permutations of residuals under a reduced Type III (partial) model. Permutational post hoc $t$-tests were also used where significant effects were detected. Similarity percentage (SIMPER) tests were used to identify individual fatty acid contributions to average dissimilarities among groups. All 25 identified fatty acids were used in multivariate analyses which were performed using PRIMER6 and its PERMANOVA+ (Anderson et al. 2008) software packages.

\section{RESULTS}

\section{Total lipid content — nutritional and feeding conditions}

Total lipid content for all species and sampling months were consistently higher in the liver (means ranging between 15.7 and $46.4 \mathrm{\mu g} \mathrm{mg}^{-1} \mathrm{ww}$ ) than the muscle (6.4 to $28.5 \mathrm{\mu g} \mathrm{mg}^{-1} \mathrm{ww}$ ). Total lipids in both tissues varied considerably between and within species with the lowest and highest values being recorded in sardine collected in March and November, 
respectively (Table 1). Total lipid content of sardine muscle and liver significantly differed between sampling months $\left(F_{2,25.6}=4.6, \mathrm{p}=0.001\right)$, with lower lipid content observed in tissues sampled in March, followed by July then November (Table 1). For anchovy, significantly lower lipid content was recorded in May than March and July $(p<0.002)$ and similar lipid content was observed in March and July. In March, higher muscle and liver lipid content was observed in sprat than in anchovy and sardine. In July, muscle and liver lipid content was significantly higher in sardine than anchovy $(\mathrm{p}<0.004)$.

\section{Lipid class composition - energy allocation patterns}

The dominant lipid class (as \% of total lipids) in both muscle and liver was either PL or TAG and was highly variable across species and seasons (Table 1). For both anchovy and sardine, relative levels of TAG were higher in July and November while PL was highest in March. PL in the muscle and liver was more evenly distributed over the sampling period in anchovy (means ranging between 62 and $89 \%$ ) than sardine (12 to $91 \%$; Table 1 ). In all sampling periods, with the exception of sardine sampled in November and anchovy sampled in July, ST and AMPL were higher in the liver than muscle. Ratios of TAG/ST were typically higher in muscle than liver, with the exception of anchovy sampled in March. For most lipid classes, muscle and liver showed similar temporal differences. The exception was the relative levels of SE-WE, which in sardine were highest in the liver in July (during the peak somatic growth period for sardine) and in the muscle in November (early reproduction period; Table 1). An opposite trend was apparent for anchovy, where the highest muscle SE-WE was observed in July (peak reproduction period for anchovy) and highest liver SE-WE in May (early reproduction). Another exception in anchovy showed the highest liver TAG and lowest liver PL in

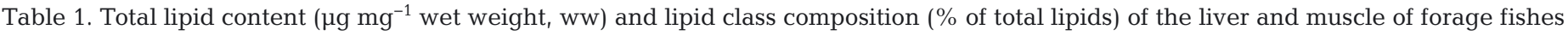
sampled in the Gulf of Lions (NW Mediterranean Sea). Values are reported as mean \pm standard deviation. SE-WE $=$ wax esters; KET $=$ ketones; TAG = triglycerides; FFA = free fatty acids; $\mathrm{ST}=$ sterols; $\mathrm{AMPL}=$ acetone mobile polar lipids; $\mathrm{PL}=$ phospholipids

\begin{tabular}{|c|c|c|c|c|c|c|c|}
\hline & - Sprat —- & & - Sardine & & & - Anchovy - & \\
\hline & March 2011 & July 2010 & March 2011 & November 2011 & July 2010 & March 2011 & May 2011 \\
\hline & Winter & Summer & Winter & Autumn & Summer & Winter & Spring \\
\hline Reproductive status & Repro (late) & Resting & Repro (late) & Repro (early) & Repro (active) & Resting & Repro (early) \\
\hline Size range $(\mathrm{cm})$ & 10.1-11.1 & $8.5-14.0$ & $12.5-14.4$ & $15.0-16.8$ & $9.0-13.0$ & $10.5-15.4$ & $8.8-14.0$ \\
\hline $\mathrm{n}$ & 5 & 16 & 10 & 5 & 31 & 40 & 9 \\
\hline Sex ratio (F:M) & $0: 1$ & $0.8: 1$ & $1: 0$ & $0.3: 1$ & $0.6: 1$ & $0: 1$ & $1: 0$ \\
\hline \multicolumn{8}{|c|}{ Total lipid $\left(\mu \mathrm{g} \mathrm{mg}^{-1}, \mathrm{ww}\right)$} \\
\hline Muscle & $18.73 \pm 3.09$ & $18.21 \pm 6.70$ & $7.79 \pm 1.47$ & $28.51 \pm 8.99$ & $9.59 \pm 1.93$ & $8.19 \pm 2.63$ & $6.38 \pm 1.11$ \\
\hline Liver & $36.21 \pm 8.47$ & $36.08 \pm 12.52$ & $15.69 \pm 4.91$ & $46.35 \pm 10.16$ & $28.81 \pm 6.59$ & $26.66 \pm 11.6$ & $16.76 \pm 3.87$ \\
\hline \multicolumn{8}{|c|}{ Lipid class (\% total lipid) } \\
\hline \multicolumn{8}{|c|}{ Muscle } \\
\hline SE-WE & $0.04 \pm 0.09$ & $0.18 \pm 0.30$ & $0.27 \pm 0.28$ & $1.22 \pm 0.65$ & $0.67 \pm 1.09$ & $0.15 \pm 0.24$ & $0.27 \pm 0.42$ \\
\hline $\mathrm{KET}$ & $0.88 \pm 0.71$ & $1.36 \pm 2.92$ & $1.26 \pm 1.48$ & $0.64 \pm 0.77$ & $0.97 \pm 0.91$ & $0.87 \pm 1.15$ & $2.41 \pm 1.70$ \\
\hline TAG & $47.62 \pm 7.75$ & $58.82 \pm 17.54$ & $1.53 \pm 0.36$ & $75.85 \pm 6.87$ & $12.99 \pm 11.78$ & $5.19 \pm 6.03$ & $6.48 \pm 9.60$ \\
\hline FFA & $0.4 \pm 0.44$ & $0.29 \pm 0.41$ & $0.00 \pm 0.00$ & $0.00 \pm 0.00$ & $0.03 \pm 0.08$ & $0.04 \pm 0.13$ & $0.83 \pm 1.00$ \\
\hline ST & $0.89 \pm 0.32$ & $0.94 \pm 0.72$ & $1.79 \pm 0.19$ & $0.50 \pm 0.60$ & $2.13 \pm 0.43$ & $1.90 \pm 0.43$ & $2.02 \pm 0.40$ \\
\hline AMPL & $3.71 \pm 1.60$ & $3.33 \pm 1.13$ & $4.00 \pm 3.50$ & $9.91 \pm 6.24$ & $4.41 \pm 1.86$ & $2.83 \pm 1.98$ & $4.54 \pm 1.85$ \\
\hline $\mathrm{PL}$ & $46.46 \pm 7.54$ & $35.08 \pm 17.11$ & $91.14 \pm 4.71$ & $11.88 \pm 3.14$ & $77.41 \pm 11.43$ & $89.01 \pm 6.52$ & $83.2 \pm 10.07$ \\
\hline TAG/ST ratio & $60.5 \pm 25.1$ & $90.1 \pm 48.7$ & $0.9 \pm 0.1$ & $150.4 \pm 14.5$ & $5.91 \pm 5.49$ & $2.92 \pm 4.22$ & $3.87 \pm 6.78$ \\
\hline \multicolumn{8}{|l|}{ Liver } \\
\hline SE-WE & $0.00 \pm 0.00$ & $1.26 \pm 3.44$ & $0.23 \pm 0.03$ & $0.10 \pm 0.19$ & $0.88 \pm 0.85$ & $0.35 \pm 0.53$ & $1.51 \pm 1.73$ \\
\hline KET & $1.28 \pm 0.59$ & $3.08 \pm 5.08$ & $0.51 \pm 0.45$ & $2.00 \pm 3.10$ & $1.26 \pm 1.10$ & $0.89 \pm 0.88$ & $4.94 \pm 3.85$ \\
\hline TAG & $38.30 \pm 14.41$ & $43.81 \pm 15.69$ & $2.65 \pm 1.40$ & $48.27 \pm 25.67$ & $12.01 \pm 8.69$ & $27.00 \pm 17.18$ & $4.97 \pm 2.59$ \\
\hline FFA & $0.04 \pm 0.08$ & $0.05 \pm 0.12$ & $0.00 \pm 0.00$ & $0.00 \pm 0.00$ & $0.04 \pm 0.09$ & $0.36 \pm 0.80$ & $0.93 \pm 1.53$ \\
\hline ST & $2.99 \pm 0.44$ & $2.75 \pm 0.91$ & $3.79 \pm 1.29$ & $0.59 \pm 0.41$ & $3.97 \pm 1.17$ & $3.97 \pm 1.34$ & $3.35 \pm 0.98$ \\
\hline AMPL & $3.96 \pm 2.84$ & $3.40 \pm 3.35$ & $1.60 \pm 0.29$ & $13.57 \pm 19.67$ & $3.02 \pm 2.68$ & $4.66 \pm 2.53$ & $8.92 \pm 7.53$ \\
\hline $\mathrm{PL}$ & $53.43 \pm 10.81$ & $45.66 \pm 14.87$ & $91.22 \pm 3.26$ & $35.48 \pm 8.39$ & $78.81 \pm 10.10$ & $62.77 \pm 16.26$ & $75.37 \pm 8.03$ \\
\hline TAG/ST ratio & $13.6 \pm 6.8$ & $19.0 \pm 12.4$ & $0.7 \pm 0.1$ & $82.0 \pm 53.1$ & $3.54 \pm 3.13$ & $8.77 \pm 9.49$ & $1.45 \pm 0.61$ \\
\hline
\end{tabular}


March (peak reproduction period) and highest muscle TAG and lowest PL in July.

Clear species differences in lipid class profiles between anchovy and sardine were apparent (Table 1), with sardine having significantly higher relative levels of TAG than anchovy in July $\left(F_{1,52.5}=\right.$ 17.6, p = 0.01), with an opposite trend in March $\left(F_{1,14.9}=8.7, \mathrm{p}=0.01\right)$. Sprat had lower relative levels of PL, SE-WE and ST, and higher levels of TAG and TAG/ST ratios than sardine and anchovy in March (Table 1). Tissue lipid class profiles of sprat were more similar to those of sardine sampled in July than sardine sampled in March or November, or anchovy in any sampling period. For all species, seasons and tissues, FFA were typically $<0.5 \%$, indicating limited tissue deterioration had occurred.

\section{Fatty acid profiles — trophic relationships}

In the neutral lipid fraction, 25 fatty acids were identified in proportions $>0.5 \%$ of total fatty acids in both the muscle and liver of sprat, sardine and anchovy (Tables $2 \& 3$ ). A PCA of all the data (for the forage fish assemblage) explained $73.4 \%$ of total variation and visually demonstrates the separation of sampling months and species (Fig. 2). PC1 explained $51.3 \%$ of the data and was largely due to differences in 22:6n3, 16:1n7, 18:1n9 and 14:0, while PC2 explained $22.1 \%$ and was largely due to variations in 18:1n9, 20:5n3 and 16:0. PERMANOVA showed that much of the variability was explained by Mo (37\%), followed by Species (18\%) and Tissue (14\%) (Table 4). A mixed Month $\times$

Table 2. Fatty acid profiles (mean \% of total fatty acids \pm standard deviation) of the neutral lipids of the muscle (M) and liver (L) of sardine sampled in the Gulf of Lions. N values and biological data are included in Table 1. SFA = saturated fatty acids; MUFA = monounsaturated fatty acids; PUFA = polyunsaturated fatty acids. Fatty acid superscripts represent trophic markers:

${ }^{\mathrm{D}}$ diatoms $;{ }^{\mathrm{F}}$ dinoflagellates $;{ }^{\mathrm{C}}$ Copepods; $^{\text {TP }}$ trophic position/carnivory index; or seasonal significantly different $(\mathrm{p}<0.01)$ markers for JUL July; ${ }^{\text {MAR }}$ March; ${ }^{\text {MAY May and }}{ }^{\text {NOV }}$ November

\begin{tabular}{|c|c|c|c|c|c|c|}
\hline & \multicolumn{2}{|c|}{ July 2010} & \multicolumn{2}{|c|}{ March 2011} & \multicolumn{2}{|c|}{ November 2011} \\
\hline & M & $\mathrm{L}$ & $\mathrm{M}$ & $\mathrm{L}$ & $\mathrm{M}$ & $\mathrm{L}$ \\
\hline $14: 0^{\mathrm{D}}, \mathrm{JUL}-\mathrm{NOV}$ & $8.16 \pm 1.12$ & $6.12 \pm 1.68$ & $4.61 \pm 1.27$ & $3.08 \pm 0.98$ & $10.19 \pm 1.88$ & $7.37 \pm 1.94$ \\
\hline $15: 0$ & $0.82 \pm 0.18$ & $0.78 \pm 0.12$ & $0.26 \pm 0.41$ & $0.58 \pm 0.35$ & $0.50 \pm 0.04$ & $0.41 \pm 0.04$ \\
\hline $16: 0^{\mathrm{MAR}}$ & $19.98 \pm 1.58$ & $19.54 \pm 1.79$ & $26.90 \pm 2.28$ & $25.67 \pm 5.80$ & $20.89 \pm 1.82$ & $18.13 \pm 2.39$ \\
\hline $17: 0$ & $0.64 \pm 0.16$ & $0.84 \pm 0.19$ & $0.34 \pm 0.43$ & $0.62 \pm 0.44$ & $0.41 \pm 0.05$ & $0.43 \pm 0.05$ \\
\hline $18: 0$ & $3.05 \pm 0.80$ & $3.30 \pm 0.79$ & $6.60 \pm 1.68$ & $4.82 \pm 1.40$ & $3.87 \pm 0.88$ & $3.86 \pm 0.62$ \\
\hline $16: 1 n 9$ & $0.61 \pm 0.22$ & $0.67 \pm 0.26$ & $0.37 \pm 0.47$ & $0.60 \pm 0.46$ & $0.53 \pm 0.23$ & $0.40 \pm 0.11$ \\
\hline $16: 1 \mathrm{n} 7^{\mathrm{D}, \mathrm{JUL}-\mathrm{NOV}}$ & $11.17 \pm 2.63$ & $8.24 \pm 3.00$ & $3.47 \pm 1.43$ & $2.48 \pm 0.99$ & $11.68 \pm 0.55$ & $9.39 \pm 2.37$ \\
\hline $18: 1 \mathrm{n} 9^{\mathrm{TP}, \mathrm{NOV}}$ & $5.88 \pm 4.29$ & $7.95 \pm 4.74$ & $8.93 \pm 1.63$ & $6.63 \pm 1.62$ & $13.42 \pm 2.72$ & $10.88 \pm 1.57$ \\
\hline $18: 1 \mathrm{n} 7^{\mathrm{F}}$ & $3.06 \pm 0.45$ & $3.74 \pm 0.92$ & $1.11 \pm 1.23$ & $3.33 \pm 1.37$ & $3.35 \pm 0.31$ & $4.02 \pm 0.33$ \\
\hline $20: 1 \mathrm{n} 11^{\mathrm{C}}, \mathrm{JUL}-\mathrm{NOV}$ & $0.93 \pm 0.50$ & $1.46 \pm 0.86$ & $0.57 \pm 0.23$ & $0.61 \pm 0.38$ & $1.19 \pm 0.41$ & $0.94 \pm 0.30$ \\
\hline $20: 1 \mathrm{n} 9^{\mathrm{C}}, \mathrm{JUL}-\mathrm{NOV}$ & $0.22 \pm 0.06$ & $0.28 \pm 0.06$ & $0.13 \pm 0.22$ & $0.17 \pm 0.14$ & $0.26 \pm 0.06$ & $0.26 \pm 0.08$ \\
\hline $22: 1 \mathrm{n} 9^{\mathrm{JUL}}$ & $1.01 \pm 0.85$ & $0.65 \pm 0.54$ & $0.19 \pm 0.20$ & $0.13 \pm 0.12$ & $0.24 \pm 0.13$ & $0.17 \pm 0.08$ \\
\hline $16: 2 \mathrm{n} 6$ & $0.41 \pm 0.12$ & $0.53 \pm 0.11$ & $0.20 \pm 0.30$ & $0.14 \pm 0.24$ & $0.35 \pm 0.06$ & $0.31 \pm 0.07$ \\
\hline $18: 2 \mathrm{n} 6^{\text {JUL-MAR }}$ & $1.32 \pm 0.28$ & $1.29 \pm 0.20$ & $1.17 \pm 0.95$ & $1.72 \pm 0.25$ & $0.76 \pm 0.15$ & $0.85 \pm 0.09$ \\
\hline $18: 3 n 3^{\mathrm{A}}$ & $1.06 \pm 0.20$ & $0.88 \pm 0.26$ & $0.66 \pm 0.97$ & $2.16 \pm 1.05$ & $0.53 \pm 0.18$ & $0.56 \pm 0.09$ \\
\hline $18: 4 \mathrm{n} 3^{\mathrm{F}, \mathrm{MAR}}$ & $2.43 \pm 0.36$ & $1.78 \pm 0.72$ & $4.85 \pm 2.67$ & $3.36 \pm 1.19$ & $0.86 \pm 0.36$ & $0.78 \pm 0.15$ \\
\hline $20: 4 \mathrm{n} 6^{\mathrm{A}, \mathrm{NOV}}$ & $0.82 \pm 0.18$ & $1.33 \pm 0.21$ & $0.28 \pm 0.46$ & $1.47 \pm 0.50$ & $2.50 \pm 0.63$ & $2.72 \pm 1.05$ \\
\hline $20: 4 \mathrm{n} 3$ JUL & $0.92 \pm 0.18$ & $1.43 \pm 0.39$ & $0.44 \pm 0.42$ & $1.86 \pm 0.90$ & $0.62 \pm 0.14$ & $0.66 \pm 0.17$ \\
\hline $20: 5 n 3^{\mathrm{D}, ~ J U L}$ & $15.55 \pm 2.79$ & $15.72 \pm 3.01$ & $9.69 \pm 3.16$ & $12.88 \pm 4.01$ & $12.91 \pm 2.31$ & $13.21 \pm 1.88$ \\
\hline $21: 5 n 3$ & $0.56 \pm 0.10$ & $0.54 \pm 0.13$ & $0.54 \pm 0.33$ & $0.55 \pm 0.14$ & $0.56 \pm 0.07$ & $0.49 \pm 0.24$ \\
\hline $22: 5 n 6$ & $0.65 \pm 0.31$ & $0.69 \pm 0.17$ & $0.36 \pm 0.56$ & $0.71 \pm 0.56$ & $0.58 \pm 0.19$ & $0.76 \pm 0.17$ \\
\hline $22: 5 n 3^{\mathrm{F}, \mathrm{NOV}}$ & $1.43 \pm 0.22$ & $2.88 \pm 1.17$ & $0.31 \pm 0.49$ & $1.01 \pm 0.75$ & $2.52 \pm 0.46$ & $2.76 \pm 0.84$ \\
\hline $22: 6 n 3^{\mathrm{F}, \mathrm{MAR}}$ & $18.08 \pm 3.17$ & $18.41 \pm 3.01$ & $25.95 \pm 2.76$ & $24.54 \pm 7.44$ & $10.27 \pm 3.42$ & $19.70 \pm 4.23$ \\
\hline Me12C14:0 & $0.31 \pm 0.11$ & $0.25 \pm 0.08$ & $0.12 \pm 0.18$ & $0.15 \pm 0.16$ & $0.20 \pm 0.03$ & $0.18 \pm 0.05$ \\
\hline Me13C16:0 & $0.93 \pm 0.19$ & $0.70 \pm 0.28$ & $1.99 \pm 3.05$ & $0.75 \pm 0.94$ & $0.80 \pm 0.05$ & $0.81 \pm 0.25$ \\
\hline$\sum$ SFA & $32.65 \pm 1.84$ & $30.58 \pm 2.29$ & $38.71 \pm 2.92$ & $34.77 \pm 5.97$ & $35.86 \pm 0.90$ & $30.19 \pm 2.43$ \\
\hline$\sum$ MUFA & $22.88 \pm 4.34$ & $22.98 \pm 4.60$ & $14.75 \pm 2.94$ & $13.95 \pm 3.24$ & $30.67 \pm 3.17$ & $26.04 \pm 3.79$ \\
\hline$\sum$ PUFA & $43.22 \pm 3.97$ & $45.48 \pm 3.77$ & $34.74 \pm 4.10$ & $37.51 \pm 7.14$ & $32.47 \pm 3.87$ & $42.78 \pm 5.93$ \\
\hline $16: 1 \mathrm{n} 7 / 16: 0^{\mathrm{D}, \text { JUL-NOV }}$ & $0.57 \pm 0.17$ & $0.43 \pm 0.18$ & $0.13 \pm 0.06$ & $0.10 \pm 0.04$ & $0.56 \pm 0.04$ & $0.52 \pm 0.15$ \\
\hline $20: 5 \mathrm{n} 3 / 22: 6 \mathrm{n} 3^{\mathrm{D}, \text { JUL-NOV }}$ & $0.90 \pm 0.28$ & $0.88 \pm 0.25$ & $0.37 \pm 0.10$ & $0.55 \pm 0.14$ & $1.35 \pm 0.40$ & $0.71 \pm 0.24$ \\
\hline $18: 1 \mathrm{n} 9 / 18: 1 \mathrm{n} 7^{\mathrm{TP}}$ & $2.11 \pm 2.15$ & $2.14 \pm 1.14$ & $4.27 \pm 1.72$ & $2.25 \pm 1.03$ & $4.04 \pm 0.95$ & $2.73 \pm 0.50$ \\
\hline
\end{tabular}


Species model for fatty acids of the small fish assemblage also showed that Month was the most important factor, and that it was more important than the interaction (Table 4). Pair-wise tests indicated that the most significant differences were between May and July $(t=7.11, \mathrm{p}<0.001)$, and between anchovy and sardine $(t=3.9, \mathrm{p}<0.002$; Table 4). SIMPER showed that March and May specimens of all species had higher contributions of 22:6n3, while samples collected in November had higher relative levels of 14:0, 16:1n7 and 18:1n9 with intermediate levels observed in July. Differences between muscle and liver fatty acid profiles were also detected $(t=4.11, \mathrm{p}<0.01)$ with SIMPER analyses showing higher influences of 22:5n3, 18:3n3, 20:4n6 and 20:4n3 in liver than muscle.
Within different months, large variations in fatty acid profiles were observed between species. In March, sprat had different fatty acid profiles and niche area compared to both sardine and anchovy, while some niche overlap was observed between anchovy and sardine (Fig. 2). Pair-wise tests for March indicated significant differences between fatty acid profiles of all species; anchovy and sprat $(t=2.95, \mathrm{p}<0.001)$, sardine and sprat ( $t=2.88, \mathrm{p}=0.002)$ and anchovy and sardine $(t=1.88, \mathrm{p}=0.022)$. Sprat had higher relative levels of 18:1n9, 16:1n7 and 20:4n3 than anchovy and sardine, while differences between anchovy and sardine were related to $22: 6 n 3,16: 0,20: 5 n 3,18: 0$ and 18:1n9. In July, tissue profiles between sardine and anchovy were significantly different $(t=4.73, \mathrm{p}<0.001)$, and were mostly related to sardine having significantly higher

Table 3. Fatty acid profiles (mean \% of total fatty acids \pm standard deviation) of the neutral lipids of the muscle (M) and liver (L) of anchovy and sprat sampled in the Gulf of Lions. N values and biological data are included in Table 1. SFA = saturated fatty acids; MUFA = monounsaturated fatty acids; PUFA = polyunsaturated fatty acids. Fatty acid superscripts represent trophic markers: ${ }^{\mathrm{D}}$ diatoms; ${ }^{\mathrm{F}}$ dinoflagellates; ${ }^{\mathrm{C}}$ copepods; ${ }^{\mathrm{TP}}$ trophic position/carnivory index; or seasonal significantly different $(\mathrm{p}<0.01)$ markers for ${ }^{\text {JUL }}$ July, MAR March, MAY May and NOV November

\begin{tabular}{|c|c|c|c|c|c|c|c|c|}
\hline & \multirow{2}{*}{\multicolumn{2}{|c|}{$\begin{array}{c}\text { Sprat }- \\
\text { March } 2011\end{array}$}} & \multirow{2}{*}{\multicolumn{2}{|c|}{ July 2010}} & \multirow{2}{*}{\multicolumn{2}{|c|}{$\begin{array}{l}\text { - Anchovy - } \\
\text { March } 2011\end{array}$}} & \multirow{2}{*}{\multicolumn{2}{|c|}{ May 2011}} \\
\hline & & & & & & & & \\
\hline & M & L & $\mathrm{M}$ & $\mathrm{L}$ & M & $\mathrm{L}$ & M & $\mathrm{L}$ \\
\hline $14: 0^{\mathrm{D}, \mathrm{JUL}}$ & $4.13 \pm 0.64$ & $3.41 \pm 0.54$ & $6.74 \pm 1.09$ & $5.77 \pm 1.70$ & $4.62 \pm 0.87$ & $4.12 \pm 0.96$ & $5.30 \pm 1.14$ & $5.31 \pm 0.78$ \\
\hline $15: 0^{\mathrm{JUL}}$ & $0.63 \pm 0.06$ & $0.40 \pm 0.03$ & $1.13 \pm 0.22$ & $1.36 \pm 0.32$ & $0.88 \pm 0.49$ & $0.82 \pm 0.15$ & $0.88 \pm 0.51$ & $0.88 \pm 0.55$ \\
\hline $16: 0$ & $26.66 \pm 1.46$ & $22.96 \pm 1.78$ & $21.23 \pm 1.31$ & $22.53 \pm 3.13$ & $25.49 \pm 2.15$ & $22.37 \pm 3.48$ & $27.47 \pm 2.95$ & $23.30 \pm 3.00$ \\
\hline $17: 0$ & $0.54 \pm 0.12$ & $0.49 \pm 0.07$ & $0.96 \pm 0.16$ & $1.07 \pm 0.21$ & $0.57 \pm 0.58$ & $0.69 \pm 0.26$ & $0.13 \pm 0.40$ & $0.49 \pm 0.51$ \\
\hline 18:0 & $2.47 \pm 0.28$ & $2.64 \pm 0.18$ & $4.15 \pm 1.10$ & $3.55 \pm 0.91$ & $5.10 \pm 1.22$ & $3.28 \pm 1.03$ & $4.70 \pm 1.25$ & $5.14 \pm 1.75$ \\
\hline $16: 1 n 9$ & $0.57 \pm 0.15$ & $0.59 \pm 0.17$ & $1.22 \pm 0.21$ & $1.46 \pm 0.51$ & $0.60 \pm 0.55$ & $0.76 \pm 0.30$ & $0.21 \pm 0.28$ & $0.39 \pm 0.29$ \\
\hline $16: 1 n 7^{\mathrm{D}, \mathrm{JUL}}$ & $4.90 \pm 0.92$ & $3.20 \pm 0.19$ & $5.91 \pm 1.61$ & $6.06 \pm 2.32$ & $2.37 \pm 1.00$ & $3.19 \pm 1.18$ & $1.93 \pm 0.87$ & $3.37 \pm 1.94$ \\
\hline $18: 1 \mathrm{n} 9^{\mathrm{TP}}$ & $15.71 \pm 3.11$ & $23.64 \pm 5.70$ & $6.20 \pm 2.16$ & $6.25 \pm 2.10$ & $6.74 \pm 1.48$ & $7.49 \pm 1.60$ & $4.98 \pm 1.26$ & $7.05 \pm 1.57$ \\
\hline $18: 1 \mathrm{n} 7^{\mathrm{F}}$ & $2.52 \pm 0.31$ & $2.91 \pm 0.32$ & $2.65 \pm 0.20$ & $3.14 \pm 0.81$ & $2.20 \pm 0.87$ & $2.73 \pm 0.78$ & $1.79 \pm 0.79$ & $3.31 \pm 1.41$ \\
\hline 20:1n11 C, MAY & $0.97 \pm 0.48$ & $0.70 \pm 0.14$ & $0.64 \pm 0.30$ & $0.32 \pm 0.12$ & $0.35 \pm 0.32$ & $0.60 \pm 0.50$ & $0.79 \pm 0.46$ & $0.82 \pm 0.90$ \\
\hline $20: 1 \mathrm{n} 9^{\mathrm{C}}$ & $0.13 \pm 0.02$ & $0.12 \pm 0.03$ & $0.28 \pm 0.10$ & $0.20 \pm 0.12$ & $0.13 \pm 0.17$ & $0.39 \pm 0.82$ & $0.24 \pm 0.24$ & $0.14 \pm 0.24$ \\
\hline $22: 1 \mathrm{n} 9$ & $0.41 \pm 0.06$ & $0.44 \pm 0.09$ & $0.52 \pm 0.30$ & $0.31 \pm 0.39$ & $0.71 \pm 0.50$ & $0.43 \pm 0.23$ & $0.31 \pm 0.25$ & $0.04 \pm 0.08$ \\
\hline $16: 2 \mathrm{n} 6$ & $0.32 \pm 0.04$ & $0.39 \pm 0.07$ & $0.55 \pm 0.10$ & $0.62 \pm 0.20$ & $0.27 \pm 0.39$ & $0.70 \pm 0.35$ & $0.00 \pm 0.00$ & $0.22 \pm 0.25$ \\
\hline $18: 2 \mathrm{n} 6$ & $1.32 \pm 0.15$ & $0.88 \pm 0.09$ & $1.54 \pm 0.22$ & $1.53 \pm 0.18$ & $1.14 \pm 0.72$ & $1.62 \pm 0.39$ & $1.22 \pm 1.10$ & $2.50 \pm 0.82$ \\
\hline $18: 3 \mathrm{n} 3^{\mathrm{A}, \mathrm{MAR}}$ & $1.35 \pm 0.19$ & $0.82 \pm 0.15$ & $0.97 \pm 0.28$ & $1.04 \pm 0.23$ & $1.34 \pm 1.01$ & $2.21 \pm 0.55$ & $0.42 \pm 0.83$ & $0.48 \pm 0.71$ \\
\hline $18: 4 \mathrm{n} 3^{\mathrm{F}, \mathrm{MAR}}$ & $2.46 \pm 0.51$ & $1.12 \pm 0.26$ & $2.44 \pm 0.81$ & $2.01 \pm 0.84$ & $3.55 \pm 1.09$ & $3.46 \pm 0.96$ & $1.65 \pm 0.74$ & $1.90 \pm 0.58$ \\
\hline $20: 4 \mathrm{n} 6^{\mathrm{A}}, \mathrm{MAY}$ & $0.49 \pm 0.11$ & $0.89 \pm 0.15$ & $0.97 \pm 0.28$ & $1.39 \pm 0.47$ & $0.58 \pm 0.50$ & $1.31 \pm 0.48$ & $1.13 \pm 0.24$ & $1.76 \pm 0.94$ \\
\hline $20: 4 n 3$ & $1.57 \pm 0.41$ & $1.67 \pm 0.36$ & $0.50 \pm 0.06$ & $0.79 \pm 0.27$ & $0.79 \pm 0.49$ & $2.01 \pm 0.46$ & $0.14 \pm 0.29$ & $0.50 \pm 0.46$ \\
\hline $20: 5 n 3^{\mathrm{D}, \mathrm{JUL}}$ & $11.53 \pm 1.58$ & $10.86 \pm 1.70$ & $12.04 \pm 1.85$ & $12.44 \pm 2.25$ & $10.80 \pm 1.25$ & $13.52 \pm 1.84$ & $10.95 \pm 1.27$ & $11.36 \pm 1.91$ \\
\hline $21: 5 n 3$ & $1.74 \pm 1.16$ & $0.48 \pm 0.20$ & $0.53 \pm 0.25$ & $0.62 \pm 0.42$ & $1.34 \pm 1.31$ & $0.47 \pm 0.13$ & $0.20 \pm 0.38$ & $0.33 \pm 0.40$ \\
\hline $22: 5 n 6$ & $0.45 \pm 0.11$ & $0.51 \pm 0.09$ & $0.89 \pm 0.21$ & $0.73 \pm 0.20$ & $0.82 \pm 0.53$ & $0.80 \pm 0.19$ & $1.08 \pm 0.83$ & $1.13 \pm 1.11$ \\
\hline $22: 5 n 3^{F}$ & $0.65 \pm 0.10$ & $1.54 \pm 0.29$ & $0.95 \pm 0.23$ & $1.05 \pm 0.36$ & $0.58 \pm 0.52$ & $1.39 \pm 0.47$ & $0.22 \pm 0.44$ & $0.70 \pm 0.48$ \\
\hline $22: 6 n 3^{F, ~ M A Y ~}$ & $17.51 \pm 2.10$ & $18.61 \pm 2.25$ & $25.55 \pm 2.43$ & $24.72 \pm 5.02$ & $27.55 \pm 4.09$ & $24.66 \pm 3.51$ & $34.09 \pm 4.80$ & $28.54 \pm 5.09$ \\
\hline Me12C14:0 & $0.22 \pm 0.01$ & $0.22 \pm 0.04$ & $0.39 \pm 0.08$ & $0.39 \pm 0.10$ & $0.19 \pm 0.28$ & $0.31 \pm 0.10$ & $0.09 \pm 0.15$ & $0.13 \pm 0.19$ \\
\hline Me13C16:0 & $0.75 \pm 0.39$ & $0.55 \pm 0.14$ & $1.06 \pm 0.64$ & $0.62 \pm 0.31$ & $1.29 \pm 1.57$ & $0.65 \pm 0.20$ & $0.07 \pm 0.20$ & $0.23 \pm 0.28$ \\
\hline$\sum \mathrm{SFA}$ & $34.43 \pm 1.29$ & $29.89 \pm 1.81$ & $34.21 \pm 1.62$ & $34.29 \pm 3.60$ & $36.66 \pm 2.47$ & $31.28 \pm 4.19$ & $38.48 \pm 1.90$ & $35.10 \pm 3.69$ \\
\hline$\sum$ MUFA & $26.54 \pm 3.83$ & $31.64 \pm 5.62$ & $17.42 \pm 2.31$ & $17.75 \pm 4.39$ & $13.10 \pm 2.70$ & $15.59 \pm 2.98$ & $10.26 \pm 2.96$ & $15.11 \pm 4.58$ \\
\hline$\sum$ PUFA & $26.53 \pm 2.75$ & $26.85 \pm 3.05$ & $46.92 \pm 3.69$ & $46.95 \pm 6.83$ & $37.96 \pm 3.05$ & $38.64 \pm 4.19$ & $40.16 \pm 2.75$ & $38.06 \pm 6.73$ \\
\hline $16: 1 \mathrm{n} 7 / 16: 0^{\mathrm{D}, \mathrm{JUL}}$ & $0.18 \pm 0.03$ & $0.14 \pm 0.01$ & $0.28 \pm 0.08$ & $0.27 \pm 0.11$ & $0.09 \pm 0.04$ & $0.15 \pm 0.06$ & $0.07 \pm 0.04$ & $0.14 \pm 0.07$ \\
\hline $20: 5 n 3 / 22: 6 n 3^{\text {D, JUL }}$ & $0.66 \pm 0.06$ & $0.58 \pm 0.03$ & $0.47 \pm 0.08$ & $0.51 \pm 0.07$ & $0.40 \pm 0.08$ & $0.55 \pm 0.08$ & $0.33 \pm 0.07$ & $0.41 \pm 0.09$ \\
\hline 18:1n9/1n $7^{\mathrm{TP}, \mathrm{MAR}}$ & $6.33 \pm 1.59$ & $8.21 \pm 2.27$ & $2.34 \pm 0.79$ & $2.08 \pm 0.81$ & $3.06 \pm 0.92$ & $2.87 \pm 0.72$ & $2.66 \pm 0.43$ & $2.40 \pm 0.93$ \\
\hline
\end{tabular}




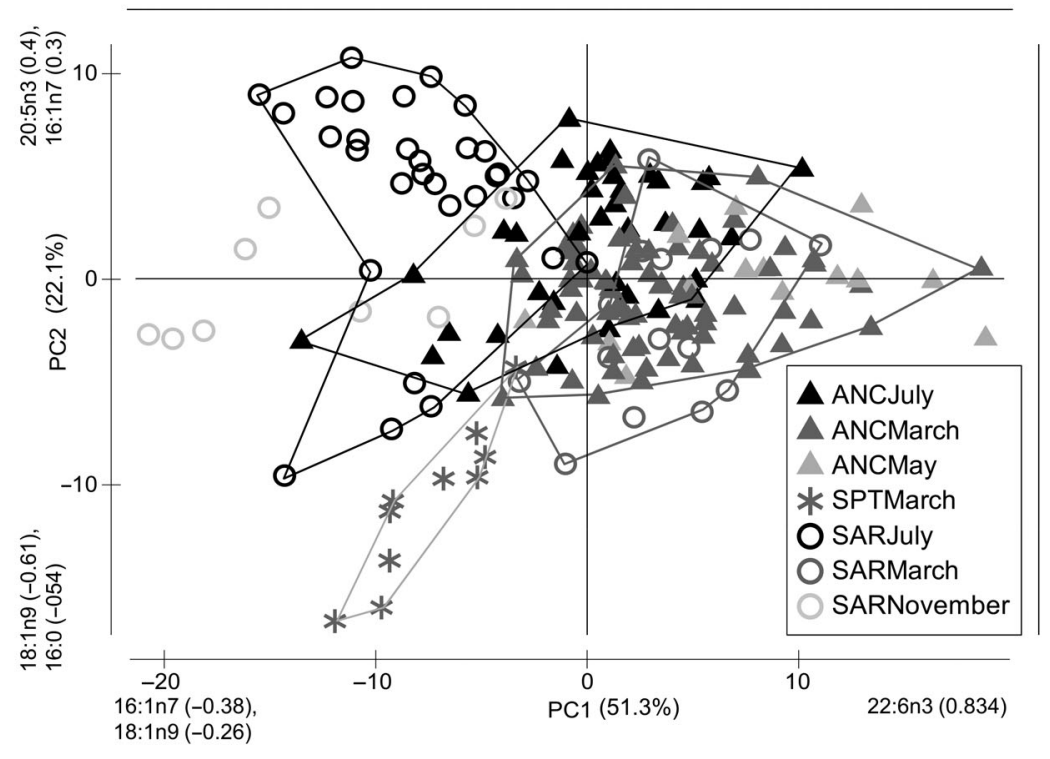

Fig. 2. Principal component analysis (PCA) of fatty acid percentage composition data for the tissues of 3 pelagic fishes (ANC, anchovy; SAR, sardine; SPT, sprat) sampled in the Gulf of Lions during summer (July 2010), autumn (November 2011), winter (March 2011) and spring (May 2011). Eigenvalues and eigenvectors are included in brackets

levels of $16: 1 \mathrm{n} 7,18: 1 \mathrm{n} 9,20: 5 \mathrm{n} 3,20: 1 \mathrm{n} 11,22: 1 \mathrm{n} 9$ and 14:0, and lower levels of 22:6n3, $16: 1 \mathrm{n} 9$ and $16: 0$ than anchovy. The overlap between the fatty acid niche areas of anchovy and sardine was more reduced in July than March (Fig. 2).
For all forage fishes combined, fatty acid markers of diatoms $(14: 0,16: 1 \mathrm{n} 7$, 20:5n3 and 16:1n7/16:0) were lower in March and May than July, and were highest in sardine collected in November (Tables $2 \& 3$ ). In contrast, markers of dinoflagellates (22:6n3 and 18:4n3) were higher in anchovy and sardine collected in March and May. Sprat sampled in March had higher fatty acid markers of carnivory (18:1n9 and 18:1n9/18:1n7) and small (Oithona and Oncaea spp.) copepods (18:1n9 and 16:0) than sardine, followed by anchovy sampled in any sampling period (Tables $2 \&$, Fig. 2). In July, sardine had higher diatom and copepod fatty acid markers than anchovy. In contrast, anchovy showed higher levels of 22:6n3, indicative of a more predominant dinoflagellatebased food chain and increased herbivory. Sardine sampled in November showed higher relative levels of copepod fatty acid markers (22:1n11 and 20:1n9) than any other period or for anchovy sampled in other months and sprat sampled in March. Algal-based fatty acid marker (18:3n3) was lowest in November sardine and March

Table 4. PERMANOVA results of the overall test for differences in fatty acid profiles (\% of total fatty acids) for all data among fixed factors: sampling period, tissue and species. Permutational post hoc t-tests were used where significant effects were detected. Significant p(MC)-values were calculated using 999 Monte Carlo permutations of residuals under a reduced and Type III (partial) model. Percent variance (\% Var) is the relative contribution of each factor to the components of variation. $\mathrm{Mo}=$ month $; \mathrm{Sp}=$ species; $\mathrm{Ti}=$ tissue $; \mathrm{Zo}=$ zone

\begin{tabular}{|c|c|c|c|c|c|c|c|c|}
\hline & \multirow[b]{2}{*}{$\mathrm{df}$} & \multirow[b]{2}{*}{ MS } & - Main test & \multirow[b]{2}{*}{$\mathrm{p}(\mathrm{MC})$} & \multirow[b]{2}{*}{$\%$ Var } & \multirow[b]{2}{*}{ Groups } & \multirow{2}{*}{$\begin{array}{c}\text {-tests } \\
t\end{array}$} & \multirow[b]{2}{*}{$\mathrm{p}(\mathrm{MC})$} \\
\hline & & & Pseudo-F & & & & & \\
\hline Mo & 3 & 2,223 & 23.67 & 0.001 & 36.83 & Jul, Mar & 5.71 & 0.001 \\
\hline \multirow[t]{5}{*}{ Residual } & 189 & 93 & & & & Jul, May & 7.11 & 0.001 \\
\hline & & & & & & Jul, Nov & 3.95 & 0.001 \\
\hline & & & & & & Mar, May & 3.69 & 0.001 \\
\hline & & & & & & Mar, Nov & 3.96 & 0.001 \\
\hline & & & & & & May, Nov & 4.74 & 0.001 \\
\hline Sp & 2 & 1292 & 11.24 & 0.001 & 18.27 & Anchovy, sardine & 3.91 & 0.001 \\
\hline \multirow[t]{2}{*}{ Residual } & 190 & 114 & & & & Anchovy, sprat & 2.91 & 0.001 \\
\hline & & & & & & Sprat, sardine & 2.22 & 0.002 \\
\hline Ti & 1 & 1984 & 16.89 & 0.001 & 14.32 & Liver, muscle & 4.11 & 0.001 \\
\hline Residual & 191 & 117 & & & & & & \\
\hline Zo & 1 & 377 & 3 & 0.032 & 2.12 & West, east & 1.73 & 0.019 \\
\hline Residual & 191 & 125 & & & & & & \\
\hline Combined model & & & & & & Jul, Mar & 6.32 & 0.001 \\
\hline Mo & 3 & 1941 & 23.18 & 0.001 & 32.82 & Jul, May & 6.45 & 0.001 \\
\hline $\mathrm{Sp}$ & 2 & 805 & 9.6 & 0.001 & 10.66 & Jul, Nov & 3.47 & 0.001 \\
\hline Mo $\times \mathrm{Sp}$ & 1 & 682 & 8.15 & 0.001 & 11.24 & Mar, May & 3.78 & 0.001 \\
\hline Residual & 186 & 84 & & & & Mar, Nov & 3.99 & 0.001 \\
\hline
\end{tabular}


Table 5. PERMANOVA results for main and post hoc $t$-test of the fatty acid profiles of (A) anchovy and (B) sardine. p(MC)-values were calculated using 999 Monte Carlo permutation of residuals under a reduced and Type III (partial) model. Percent variance $(\% \mathrm{Var})$ is the relative contribution of each factor to the components of variation. $\mathrm{Mo}=\mathrm{month} ; \mathrm{Ti}=\mathrm{tissue} ; \mathrm{Zo}=\mathrm{zone}$

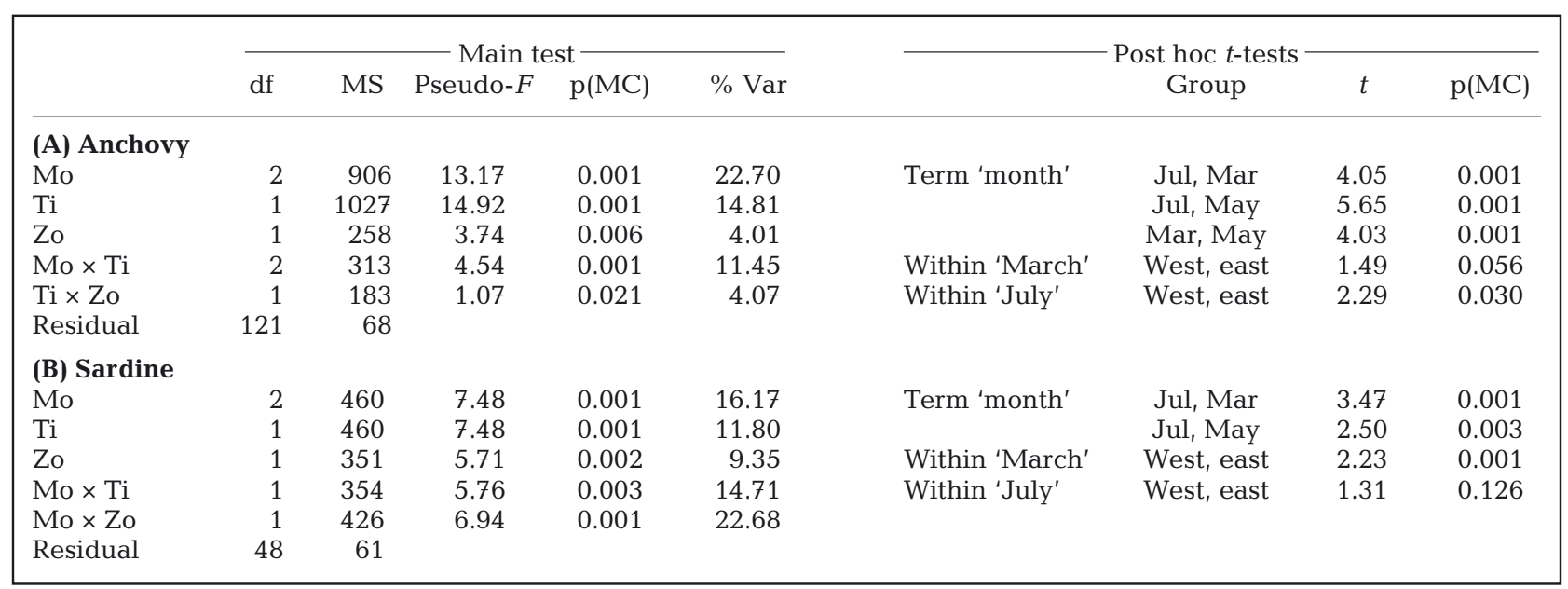

sprat and were higher in March than July for both sardine and anchovy.

Intra-specific variability in the fatty acid profiles of anchovy and sardine were also observed, and mostly

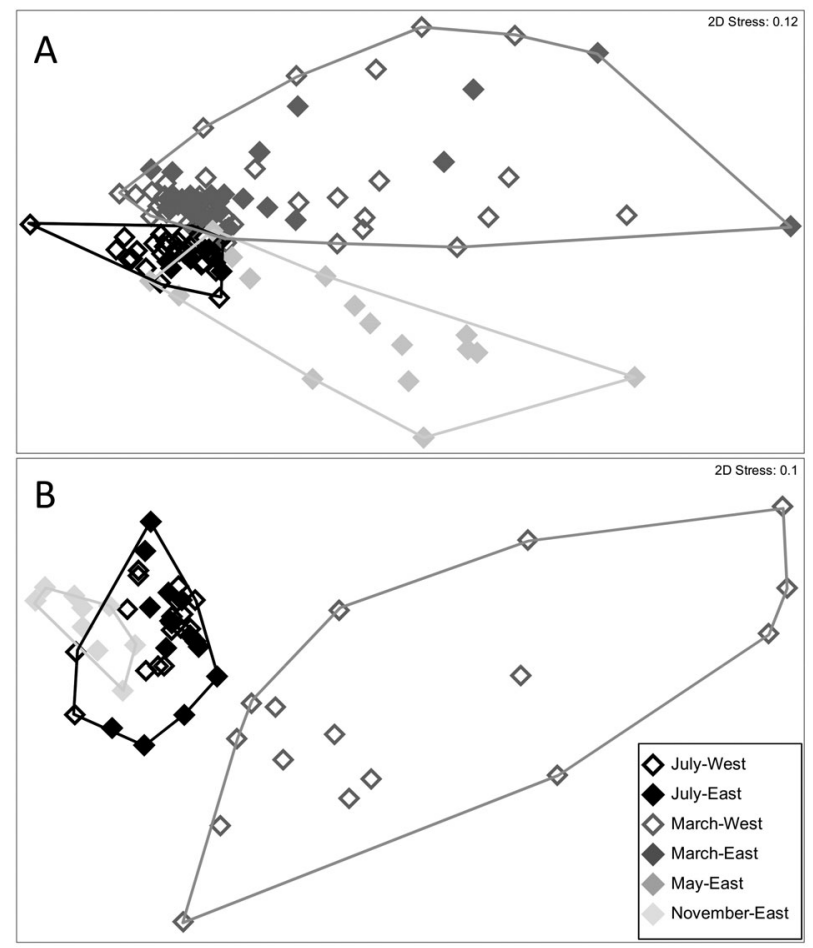

Fig. 3. Multidimensional scaling (MDS) ordinations of fatty acid percentage composition data for tissue neutral lipid fractions of (A) anchovy and (B) sardine, sampled in the west and east of the Gulf of Lions during summer (July 2010), autumn (November 2011), winter (March 2011) or spring (May 2011). Lines represent the fatty acid niche area for each sampling month related to month of sampling (Table 5, Fig. 3). For anchovy, Month as an individual factor explained more variability $(23 \%)$ than Tissue $(15 \%)$ and Zone $(4 \%)$ or any interaction with either (Table 5). For sardine, the interaction between Month and Zone explained more variation (23\%) than Month alone $(17 \%)$ or the interaction between Month and Tissue (15\%; Table 5). For both species, where tests were possible, there were no apparent effects of sex or size (PERMANOVA pseudo $F$-values $<1$, p(MC) > 0.08). An effect of sampling location was found in July for anchovy $(t=2.29, \mathrm{p}=0.003)$, and in March for sardine $(t=2.23, \mathrm{p}=0.001$; Table 5$)$. No significant differences were found between offshore and coastal areas within or among sampling months $(p>0.1)$. Among anchovy, the largest overlap in the fatty acid niche areas was observed between July and May, with limited overlap shown between March and May (Fig. 3A). Among sardine, the largest niche overlap was observed between samples caught in July and November, with neither of these months overlapping with March (Fig. 3B).

\section{DISCUSSION}

\section{Lipids infer temporal and basin-wide variations in local feeding conditions}

This study demonstrates temporal variations in feeding conditions between and among adult forage fishes collected from the NW Mediterranean Sea in summer (July 2010), winter (March 2011), spring (May 2011) and autumn (November 2011), as assessed 
by total lipid content and their components. Temporal variations in total lipid content, lipid class composition and fatty acid profiles have been reported for these and other forage fish species in the Mediterranean Sea (around northern Greece, Zlatanos \& Laskaridis 2007; Portuguese coast, Bandarra et al. 1997), and in other areas around the world (e.g. Karachi Coast, Pakistan, Nisa \& Asadullah 2008; Black Sea, Öksüz \& Ozyilmaz 2010; Baltic Sea, Peck et al. 2012, Røjbek et al. 2014). Similar differences in lipids have also been reported for other sub-temperate organisms at all levels of the trophic food chain (including low-trophic copepods, El-Sabaawi et al. 2009, Goncalves et al. 2012; mid-trophic squid, Pethybridge et al. 2013b; and high-trophic tuna, Selmi et al. 2008) and are linked to regionally specific trophic dynamics, particularly food supply (quality and quantity), which dictate the nutritional condition of consumers.

Nutritional condition of fish, as indicated by total lipid content of the muscle, was found to be highest in sardine in autumn $(\sim 2.8 \% \mathrm{ww})$ and lowest in spring for anchovy $(0.6 \%)$ and winter for sardine $(0.8 \%)$. Much better nutritional condition, but a similar temporal trend was reported for sardine fillets collected from the Portuguese coast, with total lipid content being highest in summer (18\% ww) and lowest in winter (1\%, Bandarra et al. 1997). Dissimilarity in seasonal cycles between anchovy and sardine fillets have been observed in the NE Mediterranean Sea (Zlatanos \& Laskaridis 2007) and are linked to their different feeding and energy allocation strategies and non-overlapping breeding cycles; sardine are known consumers of small zooplankton and are winter spawners, while anchovy consume larger zooplankton and are summer spawners (further discussed below).

Clear catabolic and anabolic changes in lipids have been observed in forage fish sampled more regularly. For example, in the NE Mediterranean Sea, sardine muscle was shown to have the highest lipid content in spring (11 to $12 \%$ ) and lowest in mid-winter $(4 \%)$, while anchovy and picarel fillets showed highest lipids in mid-winter to early spring (3 to 6\%) and lowest in mid-summer (1\%, Zlatanos \& Laskaridis 2007). In the Baltic Sea, total lipids and essential PUFA of the dominant forage fishes (herring and sprat) were lowest in spring ( 2 to $6 \%$ ) and highest in autumn (10 to $14 \%$, Peck et al. 2012, Røjbek et al. 2014). These large scale, cross-basin differences in total lipid content are likely related to known differences in the growth and fecundity of these populations (Silva et al. 2008), as an organism's capacity to grow, repro- duce and maintain basic life requirements is defined by its available energy.

Temporal changes in total lipid content can be related to temporal differences in local productivity and zooplankton abundances, which have been measured to be higher in summer than winter and spring in coastal areas of the GoL (Andreu \& Duarte 1996, Skovgaard \& Saiz 2006, Costalago et al. 2011) and in other areas of the NW Mediterranean (e.g. Alboran Sea, Mercado et al. 2007). However, this pattern contrasts with findings presented in other (mostly offshore) studies in the GoL where the spring-summer stratification period drives low zooplankton biomass, which is typically highest from April to June (Champalbert 1996, Gaudy \& Champalbert 1998). Interestingly, a similar but reversed trend to that typically observed in the coastal areas of the GoL was also reported for krill Meganyctiphanes norvegica in the Ligurian Sea (NW Mediterranean Sea) with highest and lowest lipid content being observed in early summer and winter, respectively (Mayzaud et al. 1999). Our findings thus suggest that (1) forage fishes sampled in this study fed close to the coast, and/or (2) the maximum chlorophyll concentrations, and thus the onset of vertical stratification of the water column which supports phytoplankton productivity, may have come later in 2010 than in previous years. Time-series data of satellite derived seasurface chlorophyll concentrations in 2010 supports the latter hypothesis of temporal changes in surface productivity (Pethybridge et al. 2013a).

\section{Lipid allocation patterns reflect different reproductive strategies and status}

In forage fish, total lipid content of individual females has been experimentally linked to recruitment strength, egg quality and quantity (Tocher et al. 1985, La Mesa et al. 2009) and consequently it has been proposed as a proxy for total egg production by fish stocks (Marshall et al. 1999). In the present study, and as shown by others (e.g. Røjbek et al. 2014), inter-specific variation in forage fish lipids can also be linked to different reproductive strategies and their related periods within a year, including spawning, summer somatic growth, and over-wintering phases (Peck et al. 2012). In the Mediterranean Sea, both sprat and sardine spawn from autumn to spring, with a peak in winter $\left(<20^{\circ} \mathrm{C}\right.$, typically in January and February, Olivar et al. 2003) and are capital breeders that rely on energy stores accumulated prior to reproduction. In contrast, anchovy spawn be- 
tween April and October, with a peak in summer (between 15 and $22^{\circ} \mathrm{C}$, typically between June and August) and are income breeders, relying on an abundant food source during their spawning phase (García \& Palomera 1996, Somarakis et al. 2004). These life-history traits and periods are reflected in the observed intra- and inter-specific differences in lipid partitioning (Table 1). For sardine, high lipid reserves in November reflect the maximum accumulated energy during summer, and are likely to indicate high recruitment strength, egg quality and quantity for the spawning season. Relatively high total lipid content in July sardine show that individuals are likely to recover from the spawning and overwintering phases and that enough energy is available to sustain somatic growth. For similar reasons, the low lipid content in anchovy in May 2011, at the start of the reproductive cycle, are likely to indicate poor recruitment and low nutritional quality of offspring, while moderate lipid levels in July 2010 indicate good potential for both growth and reproduction.

Higher lipid reserves in sardine than anchovy in July are likely related to the fact that there is an overlap between the summer somatic growth and spawning phase for anchovy. The high total lipid content in sprat in March might be seen as an indicator of better post-spawning recovery for sprat than sardine. However, given the limited sample size and the single season sampled, further studies are needed to reach such a conclusion. Since 2008, the catch abundance of sprat in the GoL has been increasing, whereas anchovy and sardine stocks have shown a decreasing trend (FAO 2011). The ability for sprat to acquire higher energy reserves during the spawning period may present a mechanism that is allowing them to challenge or outcompete sardine and anchovy, the traditionally more dominant species in the GoL. Future research should seek to explore the hypothesis that, in addition to other environmental factors, lipid-related trophic and energetic differences explain changes in forage fish species dominance in the area.

\section{Fatty acids infer temporal changes in the base of the food chain}

Fatty acid profiles reflect baseline food web dependencies (e.g. diatoms vs. dinoflagellates) and can reveal dominant food sources and degrees of carnivory (Dalsgaard et al. 2003, El-Sabaawi et al. 2009, Parrish 2013). In the GoL forage fish populations, a comparatively higher presence of dinoflagellate markers was observed in late winter (March 2011), with diatom and copepod indicators in late Autumn (November 2011), and a mixed omnivorous diet in summer (July 2010) (Fig. 1). For all seasons, ratios of 16:1n7/16:0 were lower than 3.0 indicating that they were not entirely dominated by diatoms, whereas ratios in March were $<0.3$ and clearly indicate a dominance of dinoflagellates (Dalsgaard et al. 2003). These results agree with those of Costalago et al. (2012), who found more pronounced diatom-based fatty acids in larvae of anchovy sampled in the GoL in July than December 2007. Maximum algal biomass in the NW Mediterranean Sea has been reported in the late winter/early spring mixing period (SiokouFrangou et al. 2010), and likely explains the high concentrations of algal and dinoflagellate markers in forage fishes sampled in March and May. In contrast, a predominance of diatoms is considered typical for an upwelling area (Mercado et al. 2007), and is in agreement with the high total lipid content observed during November and July.

Temporal accounts of the taxonomic composition of phytoplankton communities inhabitation in the GoL are scarce, although in the NW Mediterranean Sea, 2 well-differentiated communities have been observed over an extended temporal period: one, a diatomdominated community from 1994 to 1997 and the second, a non-siliceous algae-dominated community (characterised by the dominance of coccolithophorids and dinoflagellates) from 2000 to 2002 (Mercado et al. 2007). Our results may reflect a different annual cycling in the GoL in 2010 and 2011, which is also suggested by the forage fish lipid content data presented in this study. Such large inter-annual differences have been observed in the SW Mediterranean Sea, where high nutrient inputs and meteorological conditions in late summer contributed to the development of a large diatom bloom in autumn (DalyYahia Kéfi et al. 2005). Temporal shifts in the taxonomic composition of the phytoplankton community towards non-diatom dominated communities have been related to decreases in nitrate relative to phosphate availability (Béthoux et al. 2002).

In addition to diatom markers, fatty acid markers of large copepods (>4.1 mm, e.g. Calanus spp.) and small copepods $(<1.4 \mathrm{~mm}$, e.g. Oithona and Oncaea spp.) were highest in November sardine, suggesting a higher presence during this period. This agrees with the work of Costalago et al. (2012) who showed that copepods occupy a higher percentage composition in autumn (38\%) followed by winter (27\%) than summer (11\%). Similarly, the relatively low levels of SE-WE in all species and seasons is likely related to the fact that 
relatively few Calanus spp. (which are known to be high in SE-WE; Kattner et al. 1994) are found in the GoL (Costalago et al. 2011). In contrast, the higher indication of Oithona and Oncaea spp. in the diets of forage fishes is not surprising as they represent the dominant copepods in the GoL throughout the year (Costalago et al. 2011, Espinasse et al. 2013). The mixed diatom and dinoflagellate dietary signature in July samples agrees with a more diverse availability of plankton during this period than winter, including the additional presence of both Cladocera and Mollusca, which are limited in other seasons (Costalago et al. 2011). Fatty acid markers of euphausiids (20:5n3, $18: 4 \mathrm{n} 3$ ) were also higher in July than other sampling months, which contribute to the mixed diet signature. In agreement with the total lipid content data, relative levels of MUFA, which can be more readily catabolised to generate metabolic energy than SFA and PUFA (Sargent et al. 1999), were highest in summer than winter, reflecting better feeding conditions. This is a somewhat different trend to that shown by Garrido et al. (2008) who described a steep decrease in muscle MUFA (of the total lipid fraction) at the start of the reproductive period in sardine off Portugal.

Despite recent surveys in the GoL showing that the abundance, biomass and composition of zooplankton communities vary spatially, mainly on an onshore vs. offshore gradient (Espinasse et al. 2013), few spatial differences were observed for anchovy and sardine sampled in this study (Fig. 2). Instead, we found spatial differences in fatty acid profiles between the east and west in March for sardine, and July for anchovy (Table 5). These results suggest that forage fishes are largely consuming a spatially homogenous composition of plankton in the GoL at the time of sampling, or that they cover a large spatial area to acquire their food. The present study found no evidence that variations in lipids were related to size, sex or maturation stage, although large differences can be observed between the lipid profiles of adults and larvae (Costalago et al. 2011) sampled from the same region. Differences in lipid fraction quality with length and sex have been observed in sardine (Caponio et al. 2004).

\section{Fatty acid profiles indicate distinct trophic ecologies of forage fishes}

Fatty acid profiles of the 3 species mostly reflect omnivorous feeding, with more pronounced herbivory in March and carnivory in November (Fig. 2). Distinct inter-specific differences were identified, reflecting different feeding strategies and temporal specialisations in diets. In March, there was a higher degree of dietary overlap between sardine and anchovy, while sprat had a distinctly different diet composition with higher indicators of carnivory (18:1n9), suggesting that they are exploiting different prey species. Large dietary overlaps between anchovy and sardine and lack of overlap between sprat and sardine and sprat and anchovy have also been observed in the North Sea (Raab et al. 2012). Dietary information for sprat is currently not available in the GoL, but in the Adriatic, Black and Baltic Seas they are reported as being strict zooplanktivores, mostly feeding on copepods, followed by decapod larvae, cladocerans, invertebrate larvae and chaetognaths (Arrhenius \& Hansson 1993, Ticina et al. 2000).

In July, although there was some overlap shown in fatty acid niche areas between sardine and anchovy, sardine showed higher carnivory and higher consumption of diatoms and copepods than anchovy. These results agree with what is known of the foraging ecology of these foraging fishes. For example, in the GoL, sardine have been shown to have higher estimated trophic positions (with means ranging between 2.9 and 3.2) than anchovy (2.4 to 2.9) in a given sampling period (Costalago et al. 2012). Previous studies have also shown that sardine consume smaller zooplankton due to their filter-feeding strategy, while anchovy feed predominantly by particulate-feeding on large zooplankton (van der Lingen et al. 2006, Espinoza et al. 2009). More occurrences of diatoms in the diet composition have been reported in sardine (e.g. Demirhindi 1961, Morote et al. 2010) than anchovy (Tudela \& Palomera 1995, 1997, Plounevez \& Champalbert 2000). Such differences in the size-fraction consumed and prey preferences among sympatric forage fish species have been observed in other oceans, and are thought to be an evolutionary response to reduced species composition of prey available (Polis \& Holt 1992). Given that our findings demonstrate some different energetic pathways, the wasp-waist role of forage fishes in the GoL might be less prevalent than previously thought (Cury et al. 2000).

\section{Emphasis on analysing fatty acids of the neutral lipid fraction}

Most trophodynamics studies using signature fatty acid analyses are based on fatty acid composition of total lipids. However, depending on the tissue analysed, fatty acid compositions of total lipids can vary 
in direct relation to its lipid class composition, with tissues high in PL, such as muscle, typically having higher relative levels of PUFA, while TAG-rich tissues such as liver contain more MUFA (Pethybridge et al. 2011). These fatty acid compositional differences are related to the functional roles of these 2 dominant lipid classes, with PL playing a major structural role whereas fatty acids associated with TAG are used to store energy acquired directly from the diet (Tocher 2003). In the present study, we performed fatty acid analysis on the neutral lipid fraction only and found limited disparity between muscle and liver tissues of forage fishes (Tables 2, 4 \& 5). Furthermore, variability between these tissues is likely associated with temporal differences in diet, as liver has a faster turnover rate than that of muscle tissue. We recommend future studies planning to undertake signature fatty acid analysis consider this, and that experimental studies clarify its utilisation.

\section{Using lipids to monitor ecosystem dynamics under global change}

Global changes such as increases in temperature and declines in productivity could have a tremendous impact on the food quality and energy flux to forage fish populations and to higher-order predators, which intricately includes lipids. The Mediterranean Sea has been recognised as a 'hotspot' for climate change (Giorgi \& Lionello 2008), with predictions that temperatures will rise and plankton productivity will decline (Lionello et al. 2006, Durrieu de Madron et al. 2011). In the NW Mediterranean Sea, long-term shifts in plankton productivity and temperature have already been recorded (Marty \& Chiaverini 2002, Vandromme et al. 2010). Directly relevant to foraging fish stocks, recent reports present evidence that the GoL has shown signs of disequilibrium since 2008, with important changes in the stock structure of sardine and anchovy and an unusually high abundance of sprat (FAO 2011). As shown in this and other studies, assessment of lipid dynamics in foraging fish is an ideal way to understand variability in their population dynamics, monitor energy availability and transfer in a food web, and stipulate the flow-on effects of these observed changes to their predators, including humans. For example, in this study for both sardine and anchovy, lipid contents were indicative of fish in poor or lean nutritional condition $(<2 \%$, Nisa \& Asadullah 2008), which may be related to lower food availability and could increase their susceptibility to a range of en- vironmental stressors (e.g. predation, temperature stress, pollution). When lipid storage (indicative of enhanced feeding, reproduction and survival) conditions for forage fish are poor, fishing effort may need to be reduced to prevent population collapse. Within a management context, continuous recordings of total lipid content and fatty acid composition in key species and critical areas (such as biodiversity and climate hotspots) could be used as robust and independent indicators of habitat quality and ecosystem states; information that can be used to support an ecosystem approach to fisheries.

In a changing ocean, over short and long (climate change) time scales, long-term studies of forage fish lipids could play a role in revealing shifts in the productivity of their consumer populations, particularly where they are the most abundant mid-trophic food source. Low lipid content in forage fish is likely to have a large influence on high-order production. Several studies have connected the quality of forage fish nutrition (total lipid energy and fatty acid composition) to the physiological condition of higher-order predators (e.g. seals, Rosen \& Trites 2005, Jeanniard du Dot et al. 2008). From an energetic point of view, recent local changes in sprat abundances may represent an energetically and nutritionally better prey item than sardine and anchovy in March, whilst in July, sardines were shown to have more total lipid content but lower levels of the essential fatty acid 22:6n3 than anchovy.

\section{CONCLUSIONS}

This work is a first attempt to detail the lipid content, lipid class and fatty acid composition of adult forage fishes in the GoL, an important region commercially, ecologically and in respect to its high vulnerability to global climate change. We found clear temporal differences in the nutritional condition, energy allocation patterns and trophic interactions between and among forage fishes collected from the NW Mediterranean Sea. These differences were related to the combined effects of local feeding conditions and the life-history strategy of the species. Higher total lipid content and a diatom-based food chain signature were observed in summer and autumn, while low lipid content and dinoflagellatebased markers were more dominant in winter and spring. A high degree of dietary overlap, and thus resource competition, was observed between anchovy and sardine. Despite the small sample size, sprat did not seem to overlap with anchovy or sardine. The 
ability to detect temporal patterns of these important eco-physiological parameters is a vital link to understanding key mechanisms that drive the population dynamics of forage fishes, and which consequently affect predators and fisheries relying on these key prey species. We encourage future work to undertake lipid analysis on a larger number of samples and across a greater temporal sampling window in order to explore seasonal and annual variations and assist tactical fisheries management by providing ecosystem state indicators.

Acknowledgements. We are grateful to people who contributed to the field work, including the fisherman C. Roger and his crew on board the fishing trawler 'Roger-Christian III', as well as the scientific team and crew on board the RV 'L'Europe'. We also thank Fabrice Pernet (IFREMER) and Maria Angélica Usta-Bony (Ecole d'Ingénieur de Purpan) for their help with lipid and fatty acid analyses. The paper was improved by comments from Peter Nichols, Cathy Bulman and 3 anonymous external reviewers. This research was conducted within the project COSTAS (ANR CES AA-PPPP-007).

\section{LITERATURE CITED}

Adams SM (1999) Ecological role of lipids in the health and success of fish populations. In: Arts MT, Wainman BC (eds) Lipids in freshwater ecosystems. Springer, New York, NY, p 300-318

Anderson MJ, Gorley RN, Clarke KR (2008) PERMANOVA+ for PRIMER: guide to software and statistical methods. PRIMER-E, Plymouth

Andreu P, Duarte CM (1996) Zooplankton seasonality in Blanes Bay (northwest Mediterranean). Publ Espec Inst Esp Oceanogr 22:47-54

Arrhenius F, Hansson S (1993) Food consumption of larval, young and adult herring and sprat in the Baltic Sea. Mar Ecol Prog Ser 96:125-137

Auel H, Harjes M, da Rocha R, Stübing D, Hagen W (2002) Lipid biomarkers indicate different ecological niches and trophic relationships of the Arctic hyperiid amphipods Themisto abyssorum and T. libellula. Polar Biol 25: 374-383

Bandarra NM, Batista I, Nunes ML, Empis JM, Christie WW (1997) Seasonal changes in lipid composition of sardine (Sardina pilchardus). J Food Sci 62:40-42

Béthoux JP, Morin P, Ruiz-Pino DP (2002) Temporal trends in nutrient ratios: chemical evidence of Mediterranean ecosystem changes driven by human activity. Deep-Sea Res II 49:2007-2016

Caponio F, Lestingi A, Summo C, Bilancia MT, Laudadio V (2004) Chemical characteristics and lipid fraction quality of sardines (Sardina pilchardus W.): influence of sex and length. J Appl Ichthyology 20:530-535

Champalbert G (1996) Characteristics of zooplankton standing stock and communities in the Western Mediterranean Sea: relations to hydrology. Sci Mar 60:97-113

> Costalago D, Tecchio S, Palomera I, Álvarez-Calleja I, Ospina-Álvarez A, Raice-vich S (2011) Ecological understanding for fishery management: condition and growth of anchovy late larvae during different seasons in the Northwestern Mediterranean. Estuar Coast Shelf Sci 93:350-358

> Costalago D, Navarro J, Álvarez-Calleja I, Palomera I (2012) Ontogenetic and seasonal changes in the feeding habits and trophic levels of two small pelagic fish species. Mar Ecol Prog Ser 460:169-181

> Cury P, Bakun A, Crawford RJM, Jarre A, Quinones RA, Shannon LJ, Verheye HM (2000) Small pelagics in upwelling systems: patterns of interaction and structural changes in "wasp-waist" ecosystems. ICES J Mar Sci 57:603-618

> Dalsgaard J, St. John M, Kattner G, Muller-Navarra D, Hagen W (2003) Fatty acid trophic markers in the pelagic marine environment. Adv Mar Biol 46:225-340

Daly-Yahia Kéfi O, Soussi O, Gomez F, Daly Yahia MN (2005) Spatio-temporal distribution of the dominant diatom and dinoflagellate species in the Bay of Tunis (SW Mediterranean Sea). Mediterr Mar Sci 6:17-34

> Daskalov GM (2002) Overfishing drives a trophic cascade in the Black Sea. Mar Ecol Prog Ser 225:53-63

Demirhindi U (1961) Nutrition of the sardine (Sardina pilchardus Walb.). Proc Gen Fish Coun Med 6:253-259

- Durrieu de Madron X, Guieu C, Sempéré R, Conan P and others (2011) Marine ecosystems' responses to climatic and anthropogenic forcings in the Mediterranean. Prog Oceanogr 91:97-166

> El-Sabaawi R, Dower JF, Kainz M, Mazumder A (2009) Characterizing dietary variability and trophic positions of coastal calanoid copepods: insight from stable isotopes and fatty acids. Mar Biol 156:225-237

> Engelhard GH, Peck MA, Rindorf A, Smout SC and others (2014) Forage fish, their fisheries, and their predators: who drives whom? ICES J Mar Sci 71:90-104

Espinasse B, Devenon JL, Carlotti F, Zhou M (2013) Defining pelagic sub-regions in the Gulf of Lions (NW Mediterranean Sea) from the mesozooplankton size structure and environmental conditions. Mar Ecol Prog Ser

Espinoza P, Bertrand A, Van der Lingen CD, Garrido S, Rojas de Mendiola B (2009) Diet of sardine (Sardinops sagax) in the northern Humboldt Current system and comparison with the diets of clupeoids in this and other eastern boundary upwelling systems. Prog Oceanogr 83:242-250

FAO (2011) Report of the working group on stock assessment of small pelagic species. General fisheries commission for the Mediterranean, FAO, Rome

- Folch J, Lees M, Sloane Stanley GH (1957) A simple method for the isolation and purification of total lipids from animal tissues. J Biol Chem 226:497-509

García A, Palomera I (1996) Anchovy early life history and its relation to its surrounding environment in the Western Mediterranean basin. Sci Mar 60:155-166

Garrido S, Rosa R, Ben-Hamadou R, Cunha ME, Chícharo MA, van der Lingen CD (2008) Spatio-temporal variability in fatty acid trophic biomarkers in stomach contents and muscle of Iberian sardine (Sardina pilchardus) and its relationship with spawning. Mar Biol 154:1053-1065

Gaudy R, Champalbert G (1998) Space and time variations in zooplankton distribution south of Marseilles. Oceanol Acta 21:793-802

Gaudy R, Youssara F, Diaz F, Raimbault P (2003) Biomass, metabolism and nutrition of zooplankton in the Gulf of Lions (NW Mediterranean). Oceanol Acta 26:357-372

> Giorgi F, Lionello P (2008) Climate change projections for 
the Mediterranean region. Global Planet Change 63: 90-104

Goncalves AMM, Azeiteiro UM, Pardal MA, De Troch M (2012) Fatty acid profiling reveals seasonal and spatial shifts in zooplankton diet in a temperate estuary. Estuar Coast Shelf Sci 109:70-80

Iverson SJ, Field C, Don Bowen W, Blanchard W (2004) Quantitative fatty acid signature analysis: a new method of estimating predator diets. Ecol Monogr 74:211-235

$>$ Jeanniard du Dot T, Rosen DA, Trites AW (2008) Steller sea lions show diet-dependent changes in body composition during nutritional stress and recover more easily from mass loss in winter than in summer. J Exp Mar Biol Ecol 367:1-10

Kattner G, Wehrtmann IS, Merck T (1994) Interannual variations of lipids and fatty acids during larval development of Crangon spp. in the German Bight, North Sea. Comp Biochem Physiol 107B:103-110

Kattner G, Albers C, Graeve M, Schnack-Schiel SB (2003) Fatty acid and alcohol composition of the small polar copepods, Oithona and Oncaea: indication on feeding modes. Polar Biol 26:666-671

La Mesa M, Donato F, Giannetti G, Arneri E (2009) Growth and mortality rates of European anchovy (Engraulis encrasicolus) in the Adriatic Sea during the transition from larval to juvenile stages. Fish Res 96:275-280

> Lazzari P, Solidoro C, Ibello V, Salon S and others (2011) Seasonal and inter-annual variability of plankton chlorophyll and primary production in the Mediterranean Sea: a modelling approach. Biogeosciences Discuss 8: 5379-5422

Lionello P, Malanotte-Rizzoli P, Boscolo R, Alpert P and others (2006) The Mediterranean climate: an overview of the main characteristics and issues. In: Lionello P, Malanotte-Rizzoli P, Boscolo R (eds) Mediterranean climate variability: developments in earth and environmental sciences, Vol 4. Elsevier, Amsterdam, p 1-26

> Litzow M, Bailey K, Prahl F, Heintz R (2006) Climate regime shifts and reorganization of fish communities: the essential fatty acid limitation hypothesis. Mar Ecol Prog Ser 315:1-11

> Lloret J, Palomera I, Salat J (2004) Impact of freshwater input and wind on landings of anchovy (Engraulis encrasicolus) and sardine (Sardina pilchardus) in shelf waters surrounding the Ebre (Ebro) River delta (north-western Mediterranean). Fish Oceanogr 13:102-110

- Marshall CT, Yaragina NA, Lambert Y, Kjesbu O (1999) Total lipid energy as a proxy for total egg production by fish stocks. Nature 402:288-290

Marty JC, Chiaverini J (2002) Seasonal and interannual variations in phytoplankton production at DYFAMED time-series station, northwestern Mediterranean Sea. Deep-Sea Res II 49:2017-2030

Mayzaud P, Virtue P, Albessard E (1999) Seasonal variations in the lipid and fatty acid composition of the euphausiid Meganyctiphanas norvegica from the Ligurian Sea. Mar Ecol Prog Ser 186:199-210

> Mercado JM, Cortés D, Garcia A, Ramirez T (2007) Seasonal and inter-annual changes in the planktonic communities of the northwest Alboran Sea (Mediterranean Sea). Prog Oceanogr 74:273-293

Metcalfe LD, Schmitz AA (1961) The rapid preparation of fatty acids esters for gas chromatography analysis. Anal Chem 33:363-364

Morote E, Olivar MP, Villate F, Uriarte I (2010) A comparison of anchovy (Engraulis encrasicolus) and sardine (Sardina pilchardus) larvae feeding in the Northwest Mediterranean: influence of prey availability and ontogeny. ICES J Mar Sci 67:897-908

Nisa K, Asadullah K (2008) Seasonal variation in lipid composition of sardine (Sardinella gibbosa) from Karachi coast. J Chem Soc Pak 30:436-441

Öksüz A, Ozyilmaz KH (2010) Changes in fatty acid compositions of Black Sea anchovy (Engraulis encrasicolus L. 1758) during catching season. Turk J Fish Aquat Sci 10:381-385

Olivar MP, Catalán IA, Emelianov M, Fernández de Puelles ML (2003) Early stages of Sardina pilchardus and environmental anomalies in the northwestern Mediterranean. Estuar Coast Shelf Sci 56:609-619

Palomera I, Olivar MP, Salat J, Sabatés A, Coll M, García A, Morales-Nin B (2007) Small pelagic fish in the NW Mediterranean Sea: an ecological review. Prog Oceanogr 74:377-396

Parrish CC (2013) Lipids in marine ecosystems. ISRN Oceanogr 2013:1-16

> Peck MA, Baumann H, Bernreuther M, Clemmesen C and others (2012) The ecophysiology of Sprattus sprattus in the Baltic and North Seas. Prog Oceanogr 107:31-46

Pethybridge HR, Daley RK, Nichols PD (2011) Diet of demersal sharks and chimaeras inferred by fatty acid profiles and stomach content analysis. J Exp Mar Biol Ecol 409:290-299

Pethybridge H, Roos D, Loizeau V, Pecquerie L, Bacher C (2013a) Responses of European anchovy vital rates and population growth to environmental fluctuations: an individual-based modeling approach. Ecol Modell 250: 370-383

Pethybridge HR, Nichols PD, Virtue P, Jackson GD (2013b) The foraging ecology of an oceanic squid, Todarodes filippovae: the use of signature lipid profiling to monitor ecosystem change. Deep-Sea Res II 95:119-128

Pikitch E, Boersma PD, Boyd IL, Conover DO and others (2012) Little fish, big impact: managing a crucial link in ocean food webs. Lenfest Ocean Program, Washington, DC

Plounevez S, Champalbert G (2000) Diet, feeding behaviour and trophic activity of the anchovy (Engraulis encrasicolus L.) in the Gulf of Lions (Mediterranean Sea). Oceanol Acta 23:175-192

> Polis GA, Holt RD (1992) Intraguild predation: the dynamics of complex trophic interactions. Trends Ecol Evol 7:151-154

Raab K, Nagelkerke LAJ, Boerée C, Rijnsdorp AD, Temming A, Dickey-Collas M (2012) Dietary overlap between the potential competitors herring, sprat and anchovy in the North Sea. Mar Ecol Prog Ser 470:101-111

Razouls C, Kouwenberg JHM (1993) Spatial distribution and seasonal variation of mesozooplankton biomass in the Gulf of Lions (northwestern Mediterranean). Oceanol Acta 16:393-401

> Richoux N (2011) Trophic ecology of zooplankton at a frontal transition zone: fatty acid signatures at the subtropical convergence, Southern Ocean. J Plankton Res 33: 491-505

Røjbek MC, Tomkiewicz J, Jacobsen C, Støttrup JG (2014) Forage fish quality: seasonal lipid dynamics of herring (Clupea harengus L.) and sprat (Sprattus sprattus L.) in the Baltic Sea. ICES J Mar Sci 71:56-71

Rosen DAS, Trites AW (2005) Examining the potential for 
nutritional stress in young Steller sea lions: physiological effects of prey composition. J Comp Physiol B 175: 265-273

Rossi S, Sabatés A, Latasa M, Reyes E (2006) Lipid biomarkers and trophic linkages between phytoplankton, zooplankton and anchovy (Engraulis encrasicolus) larvae in the NW Mediterranean. J Plankton Res 28:551-562

Santojanni A, Arneri E, Bernardini V, Cingolani N, Di Marco M, Russo A (2006) Effects of environmental variables on recruitment of anchovy in the Adriatic Sea. Clim Res 31:181-193

Sargent J, McEvoy L, Estevez A, Bell G, Bell M, Henderson J, Tocher D (1999) Lipid nutrition of marine fish during early development: current status and future directions. Aquaculture 179:217-229

Selmi S, Mbarki R, Sadok S (2008) Seasonal change of lipid and fatty acid composition of little tuna Euthynnus alletteratus by-products. Nutr Health 19:189-194

Shulman GE, Nikolsky VN, Yuneva TV, Minyuk GS and others (2005) Fat content in Black Sea sprat as an indicator of fish food supply and ecosystem condition. Mar Ecol Prog Ser 293:201-212

Silva A, Carrera P, Massé J, Uriarte A, Santos MB, Oliveira PB, Soares E (2008) Geographic variability of sardine growth across the northeastern Atlantic and the Mediterranean Sea. Fish Res 90:56-69

Siokou-Frangou I, Christaki U, Mazzocchi MG, Montresor M, Ribera d'Alcalá M, Vaqué D, Zingone A (2010) Plankton in the open Mediterranean Sea: a review. Biogeosciences 7:1543-1586

Skovgaard A, Saiz E (2006) Seasonal occurrence and role of protistan parasites in coastal marine zooplankton. Mar Ecol Prog Ser 327:37-49

Somarakis S, Palomera I, Garcia A, Quintanilla L, Koutsikopoulos C, Uriarte A, Motos L (2004) Daily egg production of anchovy in European waters. ICES J Mar Sci 61:944-958

Stergiou KI, Karpouzi VS (2001) Feeding habits and trophic

Submitted: November 25, 2013; Accepted: May 14, 2014 levels of Mediterranean fish. Rev Fish Biol Fish 11:217-254

Ticina V, Vidjak O, Kacic I (2000) Feeding of adult sprat, Sprattus sprattus during spawning season in the Adriatic sea. Ital J Zool 67:307-311

Tocher DR (2003) Metabolism and functions of lipids and fatty acids in teleost fish. Rev Fish Sci 11:107-184

Tocher DR, Fraser AJ, Sargent JR, Gamble JC (1985) Lipid class composition during embryonic and early larval development in Atlantic herring (Clupea harengus, L.). Lipids 20:84-89

Tudela S, Palomera I (1995) Diel feeding intensity and daily ration in the anchovy Engraulis encrasicolus in the northwest Mediterranean Sea during the spawning period. Mar Ecol Prog Ser 129:55-61

Tudela S, Palomera I (1997) Trophic ecology of European anchovy Engraulis encrasicolus in the Catalan Sea (northwest Mediterranean). Mar Ecol Prog Ser 160: 121-134

> van der Lingen CD, Hutchings L, Field JG (2006) Comparative trophodynamics of anchovy Engraulis encrasicolus and sardine Sardinops sagax in the southern Benguela: Are species alternations between small pelagic fish trophodynamically mediated? Afr J Mar Sci 28:465-477

> Vandromme P, Stemmann L, Berline L, Gasparini S and others (2010) Zooplankton communities fluctuations from 1995 to 2005 in the Bay of Villefranche-sur-Mer (Northern Ligurian Sea, France). Biogeosciences Discuss 7:9175-9217

> Weber LP, Higgins PS, Carlson RI, Janz DM (2003) Development and validation of methods for measuring multiple biochemical indices of condition in juvenile fishes. J Fish Biol 63:637-658

Zlatanos S, Laskaridis K (2007) Seasonal variation in the fatty acid composition of three Mediterranean fish - sardine (Sardina pilchardus), anchovy (Engraulis encrasicholus) and picarel (Spicara smaris). Food Chem 103:725-728

Proofs received from author(s): August 12, 2014 